\title{
Revised distances of Northern HII regions ${ }^{\star} \star \star \star$
}

\author{
D. Russeil ${ }^{1}$, C. Adami $^{2}$, and Y. M. Georgelin ${ }^{1}$ \\ 1 LAM, 2 Place Le Verrier, 13248 Marseille Cedex 04, France \\ e-mail: delphine.russeil@oamp. fr \\ 2 LAM, Traverse du Siphon-Les trois Lucs, BP 8, 13376 Marseille Cedex 12, France
}

Received 17 July 2006 / Accepted 19 April 2007

\begin{abstract}
Aims. Our aim is to determine the distance of outer Galaxy star-forming complexes in order to model the kinematic structure of our Galaxy.

Methods. We searched for exciting star(s) of HII regions, with poor or unknown stellar distance, in the second and third galactic quadrants. We carried out spectroscopic and photometric (when necessary) observations in order to establish their spectral type and their $U, B$ and $V$ magnitudes. From these data, complemented with literature data, we determine the spectro-photometric distance of their associated complexes.

Results. We (re)established the stellar distance of 23 star forming complexes. Reinvestigating the kinematics of the Perseus and Cygnus arms, we determined the velocity departures from circular rotation and we interpreted them as streaming motions in the spiral arms. Indeed, in addition to the Perseus arm where such departures were known for a long time, we added evidence for velocity deviations in the Cygnus arm. Most significant is that we found the opposite sign for these departures in the Perseus and Cygnus arms, which suggests that the co-rotation radius is located between these two arms at $\sim 13 \mathrm{kpc}$ from the galactic center.
\end{abstract}

Key words. Galaxy: general - Galaxy: kinematics and dynamics - Galaxy: structure - ISM: HII regions

\section{Introduction}

The study of the kinematics and spiral structure of the Milky Way requires the establishment of the distance of the starforming complexes distributed into the arms. This can be done via kinematic or stellar distance determinations.

- The kinematic distance determination is based on the rotation curve of our Galaxy assuming the objects are in circular rotation. But departures from circular rotation are commonly noted inducing sometimes significant errors. In addition, these distances are unreliable toward center and anticenter directions. Finally, they depend on the solar parameters $R_{0}$ and $\theta_{0}$ which were gradually revised to lower values (Vallée 2005). This shortens the deduced kinematic distances.

- It is then essential to establish the stellar distance of the starforming complexes. This is done from the stellar distance of their constitutive HII regions. But, still today, numerous HII regions have a very uncertain distance. This is mainly due to uncertainty in, or absence of, the spectral type determination as well as to misidentification of the exciting stars.

In this framework, we have selected from the catalogue of Russeil (2003), the complexes of the second and third galactic quadrants which have uncertain or no distance determination in order to (re)establish their stellar distance.

\footnotetext{
$\star$ Based on observations collected at the Haute-Provence Observatory.

$\star \star$ Appendix A is only available in electronic form at http://www. aanda.org
}

We have focussed our study on these galactic quadrants for two reasons:

- In these quadrants the Perseus spiral arm is the dominant structural feature for which we want to determine the kinematics. Velocity anomalies in the Perseus arm have been known for a long time (Rickard 1968; Humphreys 1970, 1976; Brand \& Blitz 1993). More recently, Heyer \& Terebey (1998) show evidence that expanding motions are present within the Perseus arm.

- In the second and third quadrants we can determine the stellar distance of the Cygnus arm complexes (this is impossible in the inner galactic directions because of their larger distance and significant extinction). We want to confirm and specify the design of the Cygnus arm (=External arm = Outer arm) as well as its velocity departure we suspected in the previous paper (Russeil 2003). Indeed, it was noted that beyond the Perseus arm there are complexes which are very sparsely distributed. Several authors demonstrated the presence of far-outer Galaxy molecular material: e.g. Kutner \& Mead (1981), Digel et al. (1994, 1994), Wouterloot \& Brand (1989), Brand \& Wouterloot (1994), May et al. (1997), Heyer et al. (1998), Brunt et al. (2003), Nakagawa et al. (2005). Kaltcheva \& Hilditch (2000), from the distribution of bright $\mathrm{OB}$ stars towards longitudes $225^{\circ}$ and $245^{\circ}$ and Kimeswenger \& Weinberger (2004), from the distribution of optical tracers in the second galactic quadrant, suggested the presence of an external arm beyond the Perseus arm.

Complexes in the far-outer Galaxy are important to specify the presence and design of the Cygnus arm, in addition to trace the rotation curve at large galactocentric distance. 


\section{Observations and spectral classification}

We have selected from the catalogue of Russeil (2003), the complexes of the second and third galactic quadrants, observable from OHP (Observatoire de Haute-Provence), which have uncertain or no distance determination. To exclude local arm complexes we focus our study on complexes with kinematic distance farther than $1 \mathrm{kpc}$ and galactic latitude within $\pm 6^{\circ}$. For the complexes selected we excluded HII regions that are very extented or with very unclear morphology and/or without MSX or radio counterpart. For these HII regions the exciting star(s) are very diffult to identify.

The selected HII regions can be divided into three groups: HII regions with photometric and spectroscopic data in the literature, HII regions with only photometric data in the literature and HII regions with no data at all or very unreliable data in the literature. For the first group we directly deduced the spectrophotometric distance, for the second group we complemented the photometric data with spectroscopic observations of the exciting star candidates and finally for the third group we carried out photometric and spectroscopic observations and searched for the exciting stars candidates. In this way $32 \mathrm{HII}$ regions belonging to 23 complexes required observations.

Concerning the exciting stars themselves, we based our selection on the location of the star(s) regarding the HII region morphology (the exciting star(s) candidate(s) are expected to be strategically positioned in the HII regions seen in optical, radio and mid-IR) and from $U, B$ and $V$ photometric data (we selected the bluest candidates of the fields, from $B-V$ versus $U-B$ plots).

We limited our selection to stars brighter than $V$ magnitude 17 because we cannot observe in a reasonable exposure time the spectra of fainter stars. We then obtained the spectrum for all these candidates to deduce their spectral type.

\subsection{Photometry}

$U, B$ and $V$ images have been obtained in 2005 (1-5 Sep.) at the $1.20-\mathrm{m}$ telescope at the Observatoire de Haute Provence (OHP, France). The f/6 1.20-m OHP telescope is equipped with a thinned TK $1024 \times 1024$ pixels CCD detector, with a pixel size of 0.69 arcsec and a field of view of $11.8 \times 11.8$ arcmin. At the adopted gain, the conversion is $3.5 \mathrm{e}-/ \mathrm{adu}$, with a readout noise of $8.5 \mathrm{e}-$. The observations were calibrated and transformed into the Johnson $U B V$ system using standard stars in the catalogue of Landolt (1983). The instrumental magnitudes on the images have been measured using the Sextractor package (Bertin \& Arnouts 1996). Standard star measurements gave mean differences of $0.11 \mathrm{mag}$, that we assume as the typical uncertainty of the photometric result. This implies an uncertainty in the distance of $2.2 \%$.

\subsection{Spectroscopy}

The observations were carried out with the CARELEC spectrograph (Lemaitre et al. 1990), mounted at the Cassegrain focus of the 193-cm telescope of the OHP. The observations were carried out in three runs (16-21 Sep. 1998, 26-28 Oct. 2005 and 31 Jan.-3 Feb. 2006) using two different gratings. The gratings used give spectra with dispersion of $33 \AA / \mathrm{mm}$ and $133 \AA / \mathrm{mm}$ and a spectral range of 4055-4978 $\AA$ and 3800-6856 $\AA$ respectively. The slit is 5.5 arcmin long and has a width of 2.1 arcsec. The integration time ranged from $1200 \mathrm{~s}$ to $10800 \mathrm{~s}$ depending on the star's magnitude and the instrumental configuration. The data reduction was carried out using MIDAS. For each spectrum
Table 1. Spectral classification of known stars.

\begin{tabular}{lcc}
\hline \hline Star & $\begin{array}{c}\text { Spectral type } \\
\text { (literature) }\end{array}$ & $\begin{array}{c}\text { Spectral type } \\
\text { (found) }\end{array}$ \\
\hline Gamma Ori. & B2III & B2.5V \\
Eta Hyade & B3V & B2V \\
HD214680 & O9V & O9V \\
Upsilon Ori. & B0V & B0V \\
HD215835 & O6V & O5V \\
HD225160 & O8Ib & O7.5Ia \\
\hline
\end{tabular}

we corrected for the flat-field (tungsten lamp), bias and sky contribution. The wavelength calibration was made using an argon lamp.

\subsection{The spectral classification}

Amid the observed spectra we select the hot star candidates on the basis of the following criterion. For hot $(\mathrm{O}$ and $\mathrm{B})$ stars the primary criteria is the presence of HeII and/or HeI absorption lines. The colder stars are characterised by the absence of $\mathrm{He}$ lines but the presence of the $\mathrm{G}$ band, CaII ( $\mathrm{H}$ and $\mathrm{K}$ ) and $\mathrm{CaI}$ lines and numerous metallic lines up to $\mathrm{TiO}$ bands (for the coolest stars). The fact that the selected stars are hot is confirmed by the equivalent width of the $\mathrm{H} \gamma$ line. Following the Tables 8.1 and 9.1 of Jaschek \& Jaschek (1987) this equivalent width gives us a rough estimate of the spectral type.

To assess the spectral type we compare spectra to the spectral atlas of Walborn \& Fitzpatrick (1990) which provides lowresolution spectra in the 4000-4700 $\AA$ range. We then resized, rebined and cross-correlated (the cross-correlation was performed with IDL) the observed spectra of hot star candidates to the reference spectra allowing for possible velocity shifts between them.

The resulting data are listed in Table 2 . In this table for every HII region (listed in Col. 1) we have determined the coordinates (Cols. 3 and 4) for each observed stars from their identification on DSS images using Aladin (Bonnarel et al. 2000). Aladin (http://aladin.u-strasbg.fr/aladin.gml) is an interactive software sky atlas allowing the user to visualize digitized images of any part of the sky, to superimpose entries from astronomical catalogs or personal user data files, and to interactively access related data and information from archives for all known objects in the field. In Col. 5, we give the determined spectral type for hot stars only. For cold stars we only indicate the major features which allow us to reject them. The last column gives the alternative name of the star when available. The asterisk following some stars number means they have been observed with the lower resolution spectroscopic configuration.

To estimate the quality of our spectral type determination we observed a few stars with known spectral type in the literature. We passed them through our classification process and results are given in Table 1. Despite the small sample, we note that main sequence stars are well identified while the luminosity class is less well determined for (super)giants stars. We then adopt as typical spectral type uncertainty the maximal difference we found (one unity) and for (super)giants stars a typical difference for the luminosity class of two. On this basis, the absolute magnitude uncertainty is then deduced adopting the Mv-spectral type calibration of Russeil (2003). This uncertainty depends on the actual spectral type and luminosity class found and varies between 0.24 mag that is to say 0.07 in relative distance 
uncertainty (for a O8V) and $2.61 \mathrm{mag}$ that is to say 0.55 in relative distance uncertainty (for a B2IV).

Let us note that we took advantage of CARELEC capabilities to arrange the slit orientation to observe at all times at least two stars in each exposure, even if the second star is not a candidate.

\section{The distance of star-forming complexes}

The stellar distance of individual HII regions is calculated combining $U B V$ photometry and spectral type of the exciting stars as described in Sect. 2.2 of Russeil (2003). The results are listed in Table 3, while the identification fields and spectra of the exciting stars are presented in the online appendix.

On the basis on the new distance determination for HII regions we (re)established the distance of the star-forming complexes they belong to. A complex is the grouping of HII regions based on their kinematic and spatial nearness. The determination of the complexes and their constituting HII regions with velocities are listed and discussed in Russeil (2003, Table 1).

We also re-established the distance to the other HII regions of the complexes using spectroscopic and photometric data from the literature (using CDS/Simbad facilities). For stars with only photometric data we adopt an error on the distance of $40 \%$. For other cases the distance error is estimated in the same way as for our data. The distance of HII regions and complexes are always determined from the usual least squared method (Meyer 1975).

We discuss below the new distance determination for the complexes (summarized in Table 4 and Fig. 2). Let us note that IAU notation for Sharpless regions is "Sh2-" but for convenience in this paper we abbreviate to "S". For example Sh2-128 will be noted S128.

The complex 96.0+2.0: This complex gathers the HII regions BFS6, BFS7 and S128. Chini \& Wink (1984) established the spectral type $(\mathrm{O} 7 \mathrm{~V})$ and $U B V$ photometry of the exciting star of S128 giving a distance of $7.91 \mathrm{kpc}$. Recently, Bohigas \& Tapia (2003) reevaluated the distance (adopting the same spectral type for the star) to $9.4 \pm 0.4 \mathrm{kpc}$ using $J$ and $V$ band photometry.

We observed BFS6 but, unfortunately, no exciting star was found from spectroscopy. BFS6 has a quasi-circular $\left(17.7^{\prime} \times\right.$ $\left.16.5^{\prime}\right)$ radio counterpart, while it is a diffuse optical nebula. From our $U B V$ images (covering a large part of the radio emission extension), we made the $U-B$ versus $B-V$ plot of the field objects (Fig. 1, top). We then selected and determined the photometric distance (assuming they are main sequence stars) of the bluest stars (28 stars) brighter than $17 \mathrm{mag}$. Plotting the histogram of the distance (Fig. 1, bottom) the main feature appears as a peak at $10 \mathrm{kpc}$. This favours $9.4 \mathrm{kpc}$ for the distance of $\mathrm{S} 128$.

We then adopt for the complex a distance of $9.4 \pm 0.4 \mathrm{kpc}$.

The complex 96.3-0.2: This complex consists of BFS8 only. BSF8 is a faint nebula with a diameter of about 12' (Fich 1993). We find an $\mathrm{O} 5 \mathrm{~V}$ star as exciting star for which we established a distance of $8.8 \pm 0.61 \mathrm{kpc}$.

The complex 101.4+2.7: This complex consists only of the HII region BFS10 for which we find a stellar distance of $6.39 \pm$ $0.67 \mathrm{kpc}$. We now add the HII region DA568. Kerton et al. (2004) carried out a multiwavelength study of DA568. They estimate a mean molecular velocity of $-64 \mathrm{~km} \mathrm{~s}^{-1}$ and show
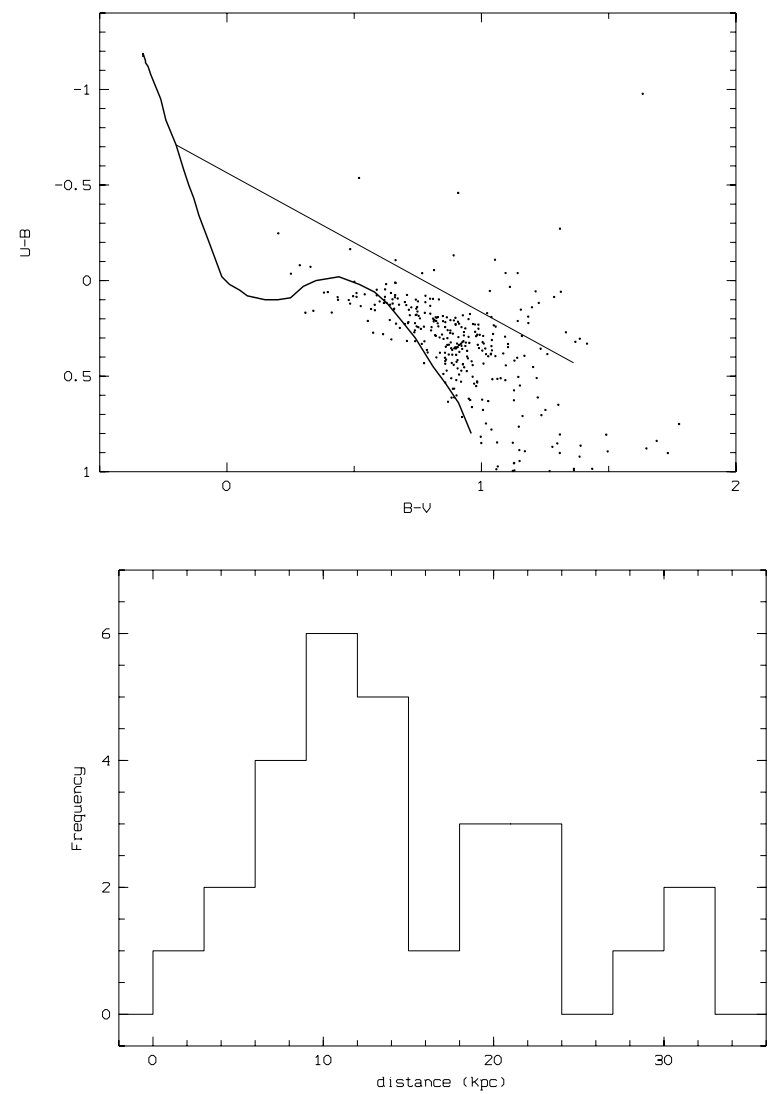

Fig. 1. Top: color diagram of stars in the field of BFS6. The curve represents the unreddened main sequence while the line is the reddening vector for a B3 star up to $A \mathrm{v}=5$ mag. Bottom: distribution of stars above the reddening vector versus the distance.

that DA 568 is the most prominent part of a much larger starforming complex that includes the optical HII region BFS 10 (considered as a blister HII region) and numerous associated IRAS sources tracing the last generation of star formation occuring in the complex. This star-forming complex appears quite evolved, with most of the parental giant molecular cloud being severely disrupted by DA 568.

It was suspected that the star ALS12050, which lies near the center of the radio emission, is a probable exciting star which we confirm from our data. We determine a distance for DA568 of $4.99 \pm 0.32 \mathrm{kpc}$ from the two stars identified.

The distance of the complex is then $5.25 \pm 0.29 \mathrm{kpc}$.

The complex 105.6+0.4: This complex is composed of S138 and S139. No stellar distance is available for S139. The spectrum of S138 stellar candidate shows emission lines, which prevents any spectral classification. Our star candidate corresponds to star 183 in Deharveng et al. (1999). From a spectrum near $\mathrm{H} \alpha$ and $8600 \AA$ they show that the star is probably a Herbig Be star. The photometric distance of the star has been evaluated in the same way as for normal stars and assuming it is a main sequence star. Indeed, Wegner (1994) shows there is no significant difference between the intrinsic flux distribution of normal $B$ and Be stars. We evaluate a distance of $2.91 \pm 1.4 \mathrm{kpc}$. We adopt this distance for this complex.

The complex 106.8+3.3: This complex consists of S141 only, for which we established a distance of $8.34 \pm 0.6 \mathrm{kpc}$. Star 1 is 
Table 2. Coordinates and spectral type of exciting star candidates.

\begin{tabular}{|c|c|c|c|c|c|}
\hline "HII region & Star number & $\begin{array}{l}\alpha_{J 2000} \\
\mathrm{~h} \mathrm{~m} \mathrm{~s}\end{array}$ & $\begin{array}{c}\delta_{J 2000} \\
0,11\end{array}$ & Spectral type & Notes \\
\hline \multirow[t]{3}{*}{ BFS6 } & 1 & 213622.1 & +522816.2 & TiO bands & \\
\hline & 2 & 213621.9 & +522720.0 & Band G & \\
\hline & 3 & 213641.7 & +522759.6 & Dominant $\mathrm{H}$ lines & \\
\hline \multirow[t]{5}{*}{ BFS8 } & 1 & 214114.2 & +523434.9 & Numerous lines & \\
\hline & 2 & 214104.6 & +523552.0 & No He lines & \\
\hline & 3 & 214103.2 & +523605.0 & Dominant $\mathrm{H}$ lines & \\
\hline & $4 *$ & 214125.1 & +523721.6 & CaII H,K lines + Band G & \\
\hline & $5 *$ & 214117.6 & +523832.3 & $\mathrm{O} 5 \mathrm{~V}$ & \\
\hline \multirow{2}{*}{ DA568 } & $1 *$ & 215515.4 & +573945.3 & $\mathrm{O} 5 \mathrm{~V}$ & ALS12050 \\
\hline & $2 *$ & 215511.4 & +573749.8 & $\mathrm{B} 2 \mathrm{~V}$ & \\
\hline BFS10 & 1 & 215630.5 & +58 0140.5 & $\mathrm{O} 9 \mathrm{~V}$ & \\
\hline \multirow[t]{3}{*}{ S138 } & 1 & 223245.5 & +582821.3 & Emission lines & \\
\hline & 2 & 223245.6 & +582737.9 & Band $\mathrm{G}+\mathrm{CaI}$ & \\
\hline & $3 *$ & 223247.7 & +582854.2 & CaII H,K lines + Band G & \\
\hline S141 & 1 & 222838.8 & +613745.3 & $\mathrm{O} 8 \mathrm{~V}$ & \\
\hline \multirow[t]{2}{*}{ S144 } & 1 & 224459.5 & +595554.3 & Band $\mathrm{G}+\mathrm{CaI}$ & \\
\hline & 2 & 224453.9 & +595104.3 & $\mathrm{TiO}$ bands & \\
\hline \multirow[t]{4}{*}{ S152 } & 1 & 225914.1 & +584431.1 & Band $\mathrm{G}+\mathrm{CaI}$, faint $\mathrm{H}$ lines & \\
\hline & 2 & 225915.3 & +58 4321.2 & Dominant $\mathrm{H}$ lines & \\
\hline & 3 & 225916.9 & +584243.4 & Band $\mathrm{G}+\mathrm{CaI}$ & \\
\hline & 4 & 225841.6 & +584656.6 & $08.5 \mathrm{~V}$ & \\
\hline S153 & 1 & 225913.6 & +584442.3 & O9V/O9.5V & ALS12719 \\
\hline S156 & 1 & 230510.3 & +601442.0 & O8 & \\
\hline \multirow[t]{3}{*}{ S158 } & 1 & 231334.3 & +613014.3 & $\mathrm{O} 9 \mathrm{~V}$ & \\
\hline & 2 & 231330.3 & +613011.6 & $\mathrm{O} 3 \mathrm{~V}$ & \\
\hline & 3 & 231321.9 & +613001.7 & No He lines & \\
\hline S159 & 1 & 231549.1 & +610758.2 & $\mathrm{O} 9 \mathrm{~V}$ & \\
\hline \multirow[t]{7}{*}{ S163 } & 1 & 233222.9 & +604005.1 & B1III & ALS13004 \\
\hline & 2 & 233230.0 & +603949.3 & Band $\mathrm{G}+\mathrm{CaI}+\mathrm{FeI}$ & \\
\hline & 3 & 233205.7 & +604041.7 & Dominant $\mathrm{H}$ lines & \\
\hline & 4 & 233208.8 & +60 4035.0 & Band $\mathrm{G}+\mathrm{CaI}$ & \\
\hline & $5^{*}$ & 233336.9 & +604506.8 & $\mathrm{O} 9 \mathrm{~V}$ & \\
\hline & $6 *$ & 233332.7 & +60 4732.1 & $\mathrm{O} 8 \mathrm{~V}$ & \\
\hline & $7 *$ & 233349.6 & +60 5107.0 & B1V & \\
\hline \multirow[t]{6}{*}{ S170 } & 1 & 000146.6 & +64 3528.7 & B1V & ALS13376 \\
\hline & $2 *$ & 000134.9 & +643724.8 & $\mathrm{O} 9 \mathrm{~V}$ & ALS13370 \\
\hline & $3 *$ & 000133.5 & +643715.6 & B2V & \\
\hline & $4 *$ & 000113.6 & +64 3519.0 & B2V & ALS13365 \\
\hline & $5^{*}$ & 000108.3 & +643721.1 & B3V & ALS13362 \\
\hline & $6^{*}$ & 000106.2 & +643724.0 & CaII $\mathrm{H}, \mathrm{K}$ lines + Band $\mathrm{G}$ & \\
\hline \multirow[t]{4}{*}{ S173 } & 1 & 002152.1 & +614527.0 & B2IV & ALS6150 \\
\hline & 2 & 002226.5 & +614939.8 & $\mathrm{~B} 1 \mathrm{~V}$ & ALS6158 \\
\hline & 3 & 002219.8 & +613909.2 & B2IV & ALS6157 \\
\hline & 4 & 002224.3 & +613923.4 & Numerous FeI lines & \\
\hline \multirow[t]{2}{*}{ S175 } & 1 & 002717.1 & +644218.0 & $\mathrm{~B} 1.5 \mathrm{~V}$ & ALS6200 \\
\hline & 2 & 002721.0 & +64 4238.1 & Band G & \\
\hline \multirow[t]{4}{*}{ S182 } & 1 & 005006.0 & +644533.5 & Emission P Cygni lines & \\
\hline & 2 & 005005.6 & +644558.2 & Band $\mathrm{G}+\mathrm{CaI}$ & \\
\hline & 3 & 005009.6 & +64 4528.1 & Band $\mathrm{G}+\mathrm{CaI}+\mathrm{FeI}$ & \\
\hline & 4 & 005003.9 & +64 4600.4 & Band G & \\
\hline \multirow[t]{3}{*}{ S187 } & 1 & 012307.3 & +615153.2 & $\mathrm{B} 2.5 \mathrm{~V}$ & \\
\hline & 2 & 012308.4 & +615134.0 & Dominant $\mathrm{H}$ lines & \\
\hline & 3 & 012312.5 & +615033.1 & Emission lines & \\
\hline \multirow{2}{*}{ S192 } & 1 & 024724.4 & +615448.0 & $\mathrm{B} 2.5 \mathrm{~V}$ & \\
\hline & 2 & 024718.2 & +615740.1 & Band $\mathrm{G}+\mathrm{CaI}+\mathrm{FeI}$ & \\
\hline \multirow{4}{*}{ S193 } & 1 & 024742.0 & +615828.1 & $\mathrm{~B} 2.5 \mathrm{~V}$ & \\
\hline & 2 & 024746.8 & +6158 17.6 & Dominant $\mathrm{H}$ lines & \\
\hline & 3 & 024740.0 & +615832.7 & $\mathrm{B} 1.5 \mathrm{~V}$ & \\
\hline & 4 & 024734.4 & +615840.4 & Faint $\mathrm{H}$ lines, No He lines & \\
\hline
\end{tabular}

well centered on the optical emission as well as on the ring-like features traced by MSX-bandA emission supporting our identification of the exciting star.
The MSX (mid-infrared Midcourse Space Experiment) band $\mathrm{A}(\lambda=8.28 \mu \mathrm{m}$ and 20 arcsec resolution) data (Price et al. 2001) span a wavelength range of $6.8-10.8 \mu \mathrm{m}$, which includes 
Table 2. continued.

\begin{tabular}{|c|c|c|c|c|c|}
\hline 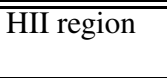 & Star number & $\begin{array}{l}\alpha_{J 2000} \\
\mathrm{~h} \mathrm{~m} \mathrm{~s}\end{array}$ & $\begin{array}{c}\delta_{J 2000} \\
{ }^{\prime}, 1\end{array}$ & Spectral type & Notes \\
\hline $\mathrm{G} 137.8+1.0$ & 1 & 024922.3 & +582643.7 & O9V & GSC03712-01870 \\
\hline \multirow[t]{4}{*}{ S196 } & 1 & 025132.2 & +62 1321.9 & Band $\mathrm{G}+\mathrm{CaI}$ & \\
\hline & 2 & 025132.1 & +62 1225.7 & Intense $\mathrm{CaI}$, faint $\mathrm{H}$ lines & \\
\hline & 3 & 025130.8 & +62 1107.0 & Band $\mathrm{G}+\mathrm{CaI}$ & \\
\hline & $4 *$ & 025132.0 & +621319.7 & $09.5 \mathrm{~V}$ & \\
\hline \multirow[t]{3}{*}{ S203 } & 1 & 032303.0 & +544716.1 & Band $\mathrm{G}+\mathrm{CaI}$ & \\
\hline & 2 & 032249.4 & +544532.9 & Band $\mathrm{G}+\mathrm{CaI}$ & \\
\hline & $3 *$ & 032037.2 & +545408.3 & (B2V phot) & \\
\hline BFS31 & $1 *$ & 032451.2 & +545705.0 & $\mathrm{B} 2 \mathrm{~V}$ & \\
\hline S204 & 1 & 035641.0 & +571529.0 & O9V & ALS7811 \\
\hline \multirow[t]{3}{*}{ S217 } & 1 & 045851.5 & +475919.6 & CaII H,K lines + Band G & \\
\hline & 2 & 045845.1 & +475954.0 & $09.5 \mathrm{~V}$ & ALS8107 \\
\hline & 3 & 045832.8 & +480104.8 & CaII H,K lines + Band G & \\
\hline \multirow[t]{7}{*}{ S219 } & 1 & 045607.1 & +472303.1 & $\mathrm{B} 2.5 \mathrm{~V}$ & \\
\hline & 2 & 045600.3 & +472242.0 & Noisy & \\
\hline & 3 & 045620.2 & +472343.0 & Band $\mathrm{G}+\mathrm{CaI}$ & \\
\hline & 4 & 045610.2 & +472332.2 & B1V & ALS8094 \\
\hline & 5 & 045608.9 & +472353.9 & Band $\mathrm{G}+\mathrm{CaI}+\mathrm{FeI}$ & \\
\hline & 6 & 045608.1 & +472401.8 & Band $\mathrm{G}+\mathrm{CaI}$ & \\
\hline & 7 & 045607.4 & +472355.3 & $\mathrm{B} 2.5 \mathrm{~V}$ & \\
\hline \multirow[t]{3}{*}{ S256 } & $1 *$ & 061236.6 & +175625.3 & CaII $\mathrm{H}, \mathrm{K}$ lines & \\
\hline & $2 *$ & 061236.6 & +175653.4 & $\mathrm{B} 2.5 \mathrm{~V}$ & \\
\hline & $3 *$ & 061236.6 & +175752.8 & CaII $\mathrm{H}, \mathrm{K}$ lines + Band $\mathrm{G}$ & \\
\hline \multirow[t]{2}{*}{ S258 } & $1 *$ & 061327.6 & +175431.6 & CaII H,K lines & \\
\hline & $2 *$ & 061327.6 & +175520.9 & B3V & \\
\hline \multirow[t]{5}{*}{ S283 } & $1 *$ & 063812.4 & +00 4400.9 & $\mathrm{~B} 3 \mathrm{~V}$ & \\
\hline & $2 *$ & 063813.6 & +00 4409.9 & $\mathrm{O} 7 \mathrm{~V}$ & \\
\hline & $3 *$ & 063815.6 & +004418.5 & $\mathrm{CaII} \mathrm{H}, \mathrm{K}$ lines $+\mathrm{Band} \mathrm{G}+\mathrm{CaI}$ & \\
\hline & $4 *$ & 063828.1 & +004440.6 & Noisy, Refilled H lines & \\
\hline & $5 *$ & 063827.3 & +004438.1 & B1V & \\
\hline BFS53 & $1 *$ & 064436.4 & +010754.6 & $\mathrm{B} 2 \mathrm{~V}$ & \\
\hline \multirow[t]{2}{*}{ S294 } & $1 *$ & 071633.2 & -092525.6 & $\mathrm{~B} 1.5 \mathrm{~V}$ & \\
\hline & $2 *$ & 071634.5 & -09 2700.4 & FeI + numerous lines & \\
\hline
\end{tabular}

Note: the asterisk following some star's number means they have been observed with the lower resolution spectroscopic configuration. ALS is acronym for Alma Luminous Star (Reed 1998).

emission bands attributed to Polycyclic Aromatic Hydrocarbons (PAHs) and thermal emission of dust (Cohen \& Green 2001). In the star-forming regions PAHs emission is a good tracer of the photon-dominated regions (Leger \& Puget 1984). The photon dominated region allows us to establish the location of the parental molecular cloud. This complements the HII region extension helping us to locate the exciting star(s).

In addition, we selected from color-color plot the bluest stars (31 stars) and determined their photometric distance assuming they are main-sequence stars. The mean value of the distribution gives a distance of $9.45 \mathrm{kpc}$. This supports the distance found for S141.

The complex 107.7+0.8: This complex consists of S144 only. We do not identify the exciting star. The DSS image shows only a faint emission with a filament-like structure. The identification is also difficult because there is no detectable MSX-Band A counterpart.

The complex 108.8-1.0: This complex is composed of S152 and S153. S152 is a bright and compact HII region excited by star 4 (distance $2.39 \pm 0.21 \mathrm{kpc}$ ) while $\mathrm{S} 153$ is a diffuse region excited by ALS12719 (distance $4.73 \pm 0.6 \mathrm{kpc}$ ). We note a large discrepancy between the distance determination of these two HII regions while Crampton et al. (1988) found them at similar distances of 3.6 and $4 \mathrm{kpc}$ respectively for $\mathrm{S} 152$ and S153. However, kinematic studies (Mirzoyan 1994; Pismis 1990; Pismis \& Hasse 1980) suggest that the two nebulae are physically linked in the way they were formed within the same cloud; but they are at different evolutionary stages, $\mathrm{S} 152$ being the youngest. The young age of S152 is confirmed by its intense MSX-Band A counterpart.

For the distance determination of the complex, we favour the distance of star 4 . Indeed, star 4 is obviously embedded in the optical emission of S152 while we can not rule out, due to the faint optical emission of S153, the possibility that ALS12719 is a field star and the possibility that $\mathrm{S} 153$ could be interpreted as a flow of ionized matter from $\mathrm{S} 152$.

The complex 110.1+0.0: This complex gathers several HII regions: BFS14, BFS15, BFS16, BFS17, BFS18 and S156.

The spectral type of the exciting star of S156 was not determined from the cross-correlation method because of the strong refilling in the hydrogen lines. The presence of HeII lines is characteristic of O type star. We estimated a O8V spectral type from the criteria (developed by Conti \& Underhill 1988) based on the equivalent widths of HeI4471 and HeII4541. Then, we 
Table 3. Photometry, distance and extinction of the exciting stars.

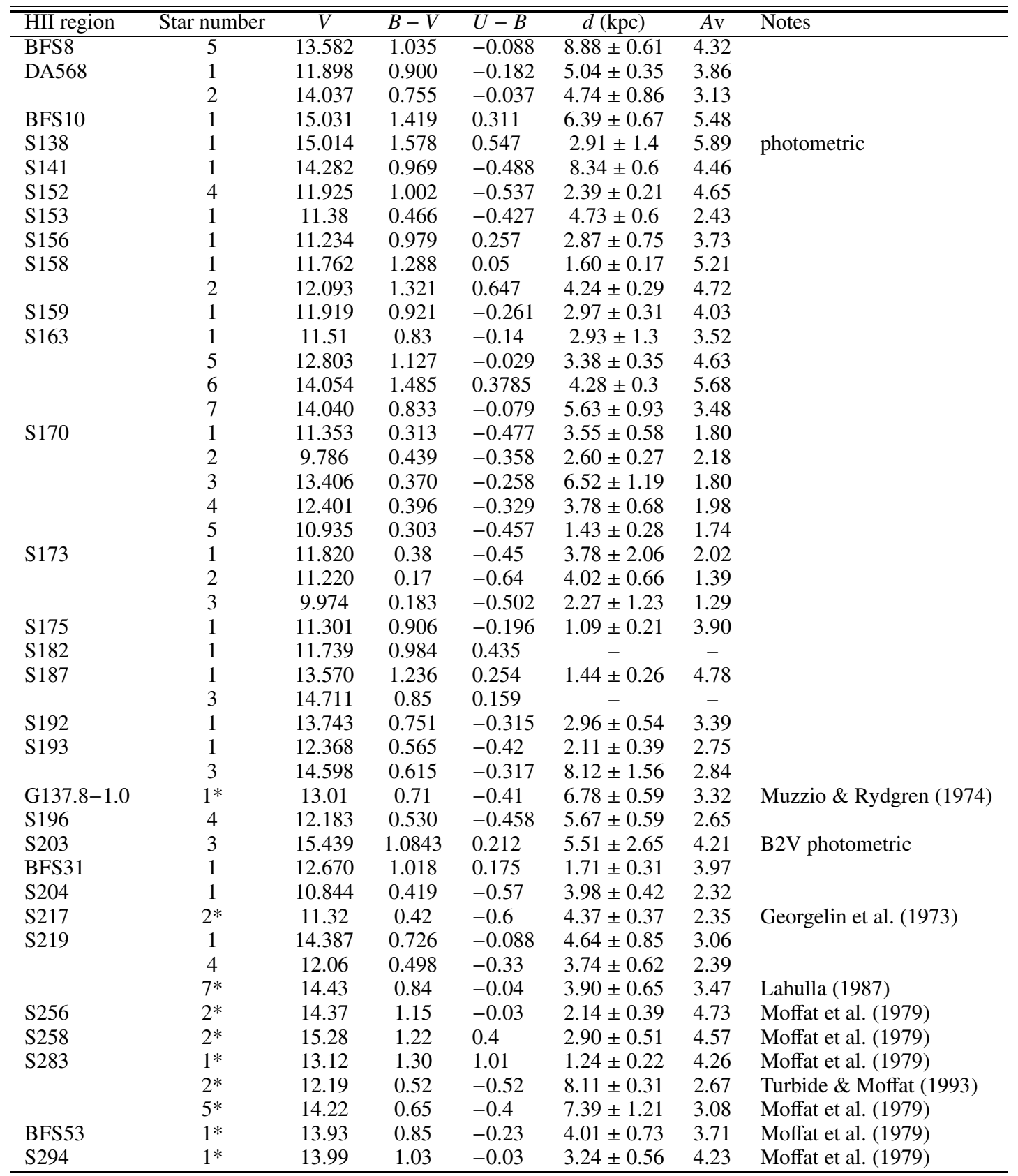

Notes: The asterisk following some star's number means that the photometry is coming from the literature whith reference in Col. 8. In Col. 6, the distance and its uncertainty (calculated from errors on the photometry, spectral type and luminosity class determination) is given.

determine the distance of $2.87 \pm 0.75 \mathrm{kpc}$ assuming the star is a main sequence star.

From CDS/ALADIN facilities we can identify the star WRAM17 (Wramdemark 1981) as a probable exciting star of BFS17. From their photometric data (Wramdemark 1981) and assuming it is a main sequence star, we estimate $\mathrm{B} 1.5 \mathrm{~V}$ as the spectral type and a distance of $3.66 \pm 1.7 \mathrm{kpc}$.

We then adopt for the complex the distance $3 \pm 0.7 \mathrm{kpc}$.

The complex 111.5-0.8: This complex is composed of the HII regions $\mathrm{S} 158$ and $\mathrm{S} 159$. We established a distance of $2.27 \pm 0.15$ for S158 and $2.97 \pm 0.31 \mathrm{kpc}$ for S159 and then for the complex a distance of $2.40 \pm 0.14 \mathrm{kpc}$.
The complex 114.0-0.7: This complex is composed of S163, S164 and S166. For S166 Foster \& MacWilliams (2006) give a spectrophotometric stellar distance of $2.57 \pm 0.6 \mathrm{kpc}$ for the exciting star BD+60 2607.

S164 is excited by the B0.2III star (Negueruela \& Marco 2003) ALS13056. Using SIMBAD we collect its photometry and calculate a distance of $2.87 \pm 0.26 \mathrm{kpc}$.

For S163 we observed four hot stars. Star 1 is outside the optical and MSX emissions while star 7 is in the direction of the PDR. Stars 6 and 5 are the closest to the center of the radio source GB6B2331+6031. Then, we establish a distance of $4 \pm$ $0.22 \mathrm{kpc}$ for $\mathrm{S} 163$ based on star 5, 6 and 7 .

We then determine a distance of $3.53 \pm 0.17 \mathrm{kpc}$ for the complex. 
Table 4. Revised stellar distance of the star-forming complexes.

\begin{tabular}{lccc}
\hline \hline Complex & $\begin{array}{c}\text { Velocity } \\
V_{\text {lsr }} \\
\mathrm{km} \mathrm{s}^{-1}\end{array}$ & $\begin{array}{c}\text { Old } \\
\text { distance } \\
\mathrm{kpc}\end{array}$ & $\begin{array}{c}\text { New } \\
\text { distance } \\
\mathrm{kpc}\end{array}$ \\
\hline $96.00+2.00$ & -74.0 & $7.8 \pm 2.3$ & $9.40 \pm 0.4$ \\
$96.30-0.20$ & -54.2 & - & $8.88 \pm 0.61$ \\
$101.40+2.70$ & -62.0 & - & $5.25 \pm 0.29$ \\
$105.60+0.40$ & -52.0 & $4 \pm 1.2$ & $2.91 \pm 1.4$ \\
$106.80+3.30$ & -64.4 & - & $8.34 \pm 0.6$ \\
$108.80-1.00$ & -51.0 & $3.7 \pm 0.8$ & $2.39 \pm 0.21$ \\
$110.10+0.00$ & -52.0 & $4.2 \pm 0.9$ & $3 \pm 0.7$ \\
$111.50-0.80$ & -55.3 & $4.1 \pm 0.9$ & $2.4 \pm 0.14$ \\
$114.00-0.70$ & -44.3 & $2.4 \pm 0.4$ & $3.53 \pm 0.17$ \\
$117.60+2.30$ & -43.7 & $2.8 \pm 0.6$ & $2.6 \pm 0.27$ \\
$119.00-1.10$ & -36.6 & $2.4 \pm 0.5$ & $3.12 \pm 0.34$ \\
$120.40+2.00$ & -49.0 & $2.3 \pm 0.7$ & $1.09 \pm 0.21$ \\
$126.70-1.00$ & -13.0 & - & $1.44 \pm 0.26$ \\
$136.10+2.10$ & -46.3 & - & $2.40 \pm 0.32$ \\
$137.80-1.00$ & -102.0 & - & $6.78 \pm 0.59$ \\
$143.60-1.80$ & -34.4 & - & $1.71 \pm 0.31$ \\
$145.80+3.00$ & -26.0 & $4 \pm 1.2$ & $3.83 \pm 0.26$ \\
$159.50+2.50$ & -23.9 & $6.1 \pm 0.7$ & $4.19 \pm 0.27$ \\
$192.50-0.10$ & 7.6 & $2.3 \pm 0.3$ & $2.46 \pm 0.16$ \\
$210.60-1.40$ & 22.0 & $1.9 \pm 0.4$ & $1.17 \pm 0.29$ \\
$211.14-1.01$ & 37.1 & - & $4.01 \pm 0.73$ \\
$213.00-1.00$ & 47.4 & $6.6 \pm 0.25$ & $7.89 \pm 0.27$ \\
$224.20+1.20$ & 33.6 & $5.4 \pm 1.6$ & $3.24 \pm 0.56$ \\
\hline
\end{tabular}

Note: the adopted error bar on the velocity $\left( \pm 5 \mathrm{~km} \mathrm{~s}^{-1}\right)$, the velocity of the complexes and the old distance are from Russeil (2003).

The complex 117.6+2.3: This complex is S170. S170 has a round (diameter $\sim 17^{\prime}$ ) and well defined morphology. All the observed stars are located in the central part of the region belonging certainly to the cluster Stock 18 . Unfortunately, no distance is available in the literature for this cluster. We found 5 stellar candidates with distance ranging from 1.4 to $6.5 \mathrm{kpc}$. Four of them are B type stars while star 2 is an $\mathrm{O}$ type star. In addition star 2 is located at the center of the HII region. We then adopt the distance of star $2(2.6 \pm 0.27 \mathrm{kpc})$ for the complex.

The complex 119.0-1.1: This complex is composed of S172, S173 and S177. No stellar distance is available for S172. For S177, Foster \& MacWilliams (2006) propose HD2421 (a B0.5IV star with distance $2.04 \pm 0.47 \mathrm{kpc}$ ) as exciting star while according to Felli \& Perinotto (1974) the exciting star is ALS6240 (a O9V star with distance $2.42 \pm 0.55 \mathrm{kpc}$ ). As S177 is an extended (diameter about $40 \mathrm{arcmin}$ ) and diffuse HII region with no clear MSX and radio counterpart, it is not possible to confirm the exciting star identification.

In the field of S173, using ALADIN we identified seven hot stars. From data extracted from the literature (Haug 1970; Nicolet 1978) we establish the distance of the stars: ALS6151 (O9V, $d=2.57 \pm 0.5 \mathrm{kpc}$ ), ALS6156 (photometric spectral type B2V, $d=4.41 \pm 1.77 \mathrm{kpc}$ ), ALS6145 (photometric spectral type B0.5V, $d=3.17 \pm 1.27 \mathrm{kpc}$ ) and ALS6155 (photometric spectral type B0.5V, $d=3.36 \pm 1.34 \mathrm{kpc}$ ). From our observations we establish the distance of the stars: ALS6150 (3.78 \pm $1.89 \mathrm{kpc}), \operatorname{ALS6158}(4.02 \pm 0.66 \mathrm{kpc})$ and ALS6157 (2.27 \pm $1.14 \mathrm{kpc})$. The distance of $\mathrm{S} 173$ is then $3.12 \pm 0.34 \mathrm{kpc}$. We adopt this distance for the complex.

The complex 120.4+2.0: This complex is S175. The star centered on the nebula is ALS6206. This star was previously

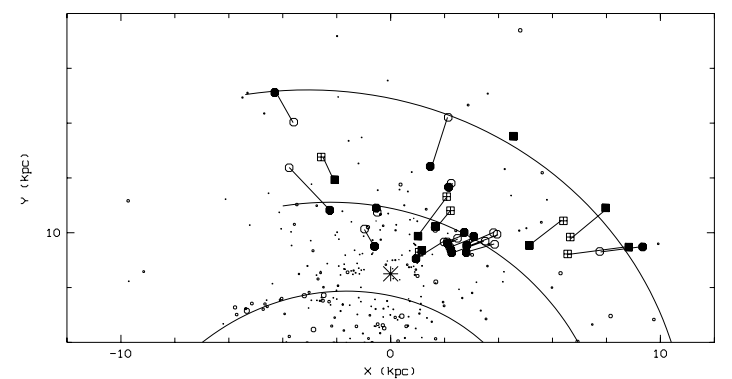

Fig. 2. New stellar distance of the complexes (filled symbols) versus old distance. The old distance is either the old stellar distance (squares) or the kinematic distance (circles). The Sun is represented by the large star symbol and the arms by the continuous lines. The curves are the fitted spiral arms from Russeil (2003).

classified as $09.5 \mathrm{~V}$ (Georgelin et al. 1973). This placed it at $2.13 \mathrm{kpc}$. Now, we find a spectral type for this star of B1.5V giving a distance of $1.09 \pm 0.21 \mathrm{kpc}$.

The complex 122.7+2.1: This complex consists of S181 and S182. No distance is available for S181 which is an extended and diffuse HII region. The exciting star of S182 exhibits emission lines which prevent any classification. Indeed, the lines, especially the hydrogen ones, exhibit $\mathrm{P}$ cygni profiles or are emission lines. S182 is associated with the source IRAS $00470+6429$. This source is classified as a proto-planetary nebula and the spectral type is Ae (Kelly \& Hrivnak 2005). S182 is then removed from the complex.

The complex 126.7-1.0: This complex previously gathered the HII regions S183, S187 and S188.

S183 must be removed from this complex. Landecker et al. (1992) show that S183 corresponds to a bright rim of radio emission on the western edge of a much larger object apparently hidden by extensive obscuring material. The region is seen as a bubble blown by the stellar winds of the star(s) which excite the HII region. In addition, Landecker et al. (1992) obtained from the radio recombination line observations a velocity of $-63.5 \mathrm{~km} \mathrm{~s}^{-1}$ which is very different from the velocity of the complex $\left(-13 \mathrm{~km} \mathrm{~s}^{-1}\right)$. Fich \& Blitz (1984) measured a CO velocity at $-10.3 \mathrm{~km} \mathrm{~s}^{-1}$ in the direction of S183 while Fich et al. (1990) give a $\mathrm{H} \alpha$ velocity of $-62.6 \mathrm{~km} \mathrm{~s}^{-1}$. Similarly, Dame et al. (1987) presented maps showing clouds of CO around this velocity. Wouterloot \& Brand (1989) list three CO velocity components at $-66.4 \mathrm{~km} \mathrm{~s}^{-1}$ (the strongest, $\left.4.2 \mathrm{~K}\right)-12.6 \mathrm{~km} \mathrm{~s}^{-1}(2.4 \mathrm{~K})$ and $-5 \mathrm{~km} \mathrm{~s}^{-1}(2.8 \mathrm{~K})$. Brunt et al. (2003) find molecular clouds in the direction of S183 distributed around two main velocities: -62.4 and $-12 \mathrm{~km} \mathrm{~s}^{-1}$. They also show that the HI profile peaks at $-63.5 \mathrm{~km} \mathrm{~s}^{-1}$. These observations indicate that the molecular material around $-13 \mathrm{~km} \mathrm{~s}^{-1}$ is probably associated with absorbing material in front of S183. S183 now forms a new complex, $123.28+3.03$, with velocity $-63 \mathrm{~km} \mathrm{~s}^{-1}$.

S188 is a planetary nebulae with a distance between $600 \mathrm{pc}$ (Harris et al. 1997) and 1000 pc (Napiwotzki 2001). It is removed from the complex.

Then the distance of the complex is based on the distance of $\mathrm{S} 187$ only which is $1.44 \pm 0.26 \mathrm{kpc}$. 
The complex 136.1+2.1: We included in this complex the HII regions S192, S193 and S196 on the basis of their velocities only.

The HII regions S192 and S193 clearly define a subgroup. Indeed the MSX-band A emission appears elongated encompassing these 2 HII regions. Near the middle of these HII regions, corresponding to an MSX emission peak $(02 \mathrm{~h} 47 \mathrm{~m} 26.47 \mathrm{~s}$ $\left.+61^{\circ} 56^{\prime} 49.2^{\prime \prime}\right)$, there is the IRAS source IRAS02435+6144 and the near infrared star clusters [BDS2003]57 (Bica et al. 2003) possibly induced by the HII regions. This consolidates the fact that they are physically linked.

We establish a distance of $2.96 \pm 0.54 \mathrm{kpc}$ for $\mathrm{S} 192$ for which the quasi-circular morphology makes easy the identification of the star. For S193 the observed star 3 exhibits very different distance. Its large distance $(8.12 \mathrm{kpc})$, suggests it is a background star. The adopted distance for S193 $(2.11 \pm 0.39 \mathrm{kpc})$ is then the distance of the star 1 .

The case of S196 is more complex. On the one hand, the ionized $\left(-48.1 \mathrm{~km} \mathrm{~s}^{-1}\right.$, Fich et al. 1990) and molecular $\left(-45.1 \mathrm{~km} \mathrm{~s}^{-1}\right.$, Blitz et al. 1982) gas velocities of S196 are similar to that of S192 and S193 suggesting they are linked. On the other hand, the stellar distance assigned to S196 is $5.67 \pm$ $0.59 \mathrm{kpc}$ (star 4). This distance is different from the distance of other HII regions of this complex. Already Hunter \& Massey (1990) found a large and unreliable distance $(9 \mathrm{kpc})$ for the identified star leading them to adopt the kinematic distance. Moreover, in Digel et al. (1994), the molecular cloud associated to S192 and S193 is clearly noted (cloud 28) while S196 appears associated to a distinct fainter molecular feature making its belonging to the complex not clear.

We choose arbitrarily to calculate the distance for the complex $(2.4 \pm 0.32 \mathrm{kpc})$ based on the distances of S192 and S193 but we note that the case of S196 is not closed.

The complex 137.8-1.0: This complex corresponds to the molecular cloud denoted as Cloud 2 in the list by Digel et al. (1994).

De Geus et al. (1993) reported an extended $\mathrm{H} \alpha$ emission with the same radial velocity as Cloud $2\left(\mathrm{VLSR}=-103 \mathrm{~km} \mathrm{~s}^{-1}\right)$. They concluded that this $\mathrm{H} \alpha$ emission traces an HII region associated with Cloud 2, and they proposed an early B-type star near Cloud 2 ("MR 1": Muzzio \& Rydgren 1974) as the photoionizing source.

Smartt et al. (1996), observed and analysed high resolution spectra of MR1 using LTE atmosphere modelisation. They estimate a distance between $8.2 \mathrm{kpc}$ and $12 \mathrm{kpc}$ and a LSR velocity for the star of $-90 \pm 13 \mathrm{~km} \mathrm{~s}^{-1}$. From our spectra we established a velocity for the star of $-102.7 \pm 12 \mathrm{~km} \mathrm{~s}^{-1}$ in agreement with the nebular and molecular cloud confirming their physical link. In addition, the geometry of ionized gas, IRAS sources, NIR sources, and molecular cloud suggests that MR 1 has triggered the star formation activity in the cloud (Kobayashi \& Tokunaga 2000) confirming the link between the star, the HII region and the molecular cloud.

We established a distance of $6.78 \mathrm{kpc}$. In agreement with Smartt et al. (1996) we confirm the large discrepancy between the stellar distance and the kinematic distance $(23 \mathrm{kpc})$ which underlines the presence of non-circular motions.

The complex 143.6-1.8: This complex gathers S203 and BFS31. S203 is an extended quite diffuse HII region $\left(1.23^{\circ} \times\right.$ $1^{\circ}$ ) as seen in $\mathrm{H} \alpha$ in the VTSS survey (Dennison et al. 1997). Only the northern part of $\mathrm{S} 203$ exhibits a radio $(5 \mathrm{GHz})$ counterpart while no MSX counterpart is noted. We identify a hot star close to the radio source GB6 B0316+5439 but its spectral classification is not possible due to the refilling of the lines. In particular the $\mathrm{H} \alpha$ line is in emission while the other hydrogen lines are clearly refilled. The HeI lines at $4390 \AA, 4471 \AA$ and $4924 \AA$ are clearly noted suggesting it is a B star. Then we estimated only the photometric distance. However, due to the large extent and the morphology of S203, the association of this star with S203 is very uncertain.

BFS31 is on the border of S203. It exhibits MSX and radio counterparts suggesting it is a recent HII region. The identification of its exciting star is unambiguous, contrary to S203. We then adopt the distance of BFS31 $(1.71 \mathrm{kpc})$ for the distance of the complex.

The complex 145.8+3.0: This complex is the HII region S204. S204 is a faint and diffuse arc-like nebula extended on about 48 arcmin without MSX band A counterpart.

From CDS/ALADIN we find in the area two radio sources: RRF1509 and GB6 B0355+5658 in the direction of which there are respectively the stars ALS7833 (O7.5, Hiltner \& Johnson 1956; Nicolet $1978, d=4.21 \pm 1.77 \mathrm{kpc})$ and BD $+56^{\circ} 866(\mathrm{O} 9 \mathrm{~V}$, Hiltner \& Johnson 1956; Haug 1970, $d=3.63 \pm 0.38 \mathrm{kpc}$ ). In addition, three hot stars, with photometric data only (Haug 1970), are identified in the field: ALS7816 (photometric spectral type B0V, $d=4.49 \pm 1.35 \mathrm{kpc}$ ), ALS7815 (photometric spectral type $\mathrm{B} 1 \mathrm{~V}, d=4.33 \pm 1.3 \mathrm{kpc}$ ) and ALS7829 (photometric spectral type $\mathrm{B} 1 \mathrm{~V}, d=3.63 \pm 1.09 \mathrm{kpc}$ ). From our observations we establish a spectral type and distance for ALS7811 of O9V and $3.98 \pm 0.42 \mathrm{kpc}$.

The distance of the HII region is then $3.83 \pm 0.26 \mathrm{kpc}$.

The complex 159.5+2.5: This complex gathers S219, S217 and BFS44. For BFS44 no stellar distance is available.

The spectral type of the main exciting star (ALS8107) of S217 is O9.5V, B0V or O8V according to Georgelin et al. (1973), Moffat et al. (1979) and Chini \& Wink (1984) respectively. Deharveng et al. (2003), from the analysis of the nebular emission lines, show that S217 and S219 appear to be lowexcitation regions suggesting a B0V spectral type for the main excitating star of $\mathrm{S} 219$ and a $09.5 \mathrm{~V}$ or O9V for the exciting star of S217.

We confirm the $09.5 \mathrm{~V}$ spectral type of Georgelin et al. (1973) for the exciting star of S217 for which we determine a distance of $4.37 \pm 0.37 \mathrm{kpc}$. For S219 we establish a distance of $3.99 \pm 0.39 \mathrm{kpc}$. The distance of the complex is then $4.19 \pm 0.27 \mathrm{kpc}$.

The complex 192.5-0.1: This complex is composed of the regions S254, S255, S256, S257 and S258. Moffat et al. (1979) identified the exciting stars of these HII regions giving the spectral type and photometry (from which we establish the distance: $\mathrm{S} 254$ (HD253247, O9V, $d=2.49 \pm 0.26 \mathrm{kpc}$ ), S255 (ALS19, $\mathrm{B} 0 \mathrm{~V}, d=2.36 \pm 0.37 \mathrm{kpc})$ and S257 (HD253327, B0V, $d=$ $2.53 \pm 0.37)$ and give only photometric information for the exciting star of S256 and S258.

The spectral type found for the exciting star of S258 is in good agreement with the photometric spectral type and its distance is $2.9 \pm 0.51 \mathrm{kpc}$. The revised distance of $\mathrm{S} 256$, which is $2.14 \pm 0.39 \mathrm{kpc}$, is in better agreement with the distance of the other HII regions. Indeed, the photometric distance was unreliable $(6.8 \mathrm{kpc})$.

The distance to this complex is then $2.46 \pm 0.16 \mathrm{kpc}$. 


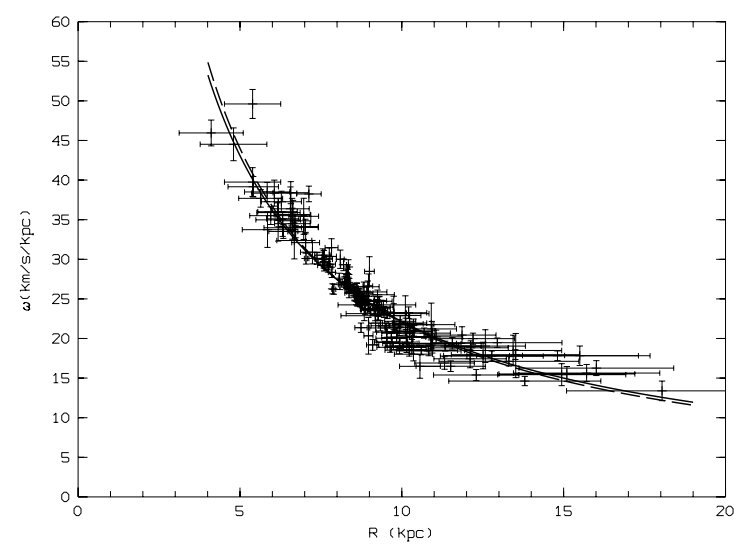

Fig. 3. Computed rotation curve (dashed line) compared with the Brand \& Blitz (1993) curve (solid line).

The complex 210.8-1.3: This complex is composed of the regions BFS53, S282 and BFS54.

The distance of BFS54 is estimated from the distance of the star HD289120. This star classified as B3 (Nesterov et al. 1995) has a distance of $1.13 \pm 0.34 \mathrm{kpc}$ assuming it is a main sequence star. The exciting star of S282 is HD47432 (Carrasco-Gonzalez et al. 2006), its spectral type is O9.5II (Karchenko 2001) and its distance is $1.3 \pm 0.55 \mathrm{kpc}$.

BFS53 was placed in this complex due to its ionised gas velocity $\left(23.5 \mathrm{~km} \mathrm{~s}^{-1}\right)$. But its $\mathrm{CO}$ velocity is $37.1 \mathrm{~km} \mathrm{~s}^{-1}$ while the velocity of S282 and BFS54 is $22 \mathrm{~km} \mathrm{~s}^{-1}$. From the stellar distance we can now put BFS53 aside. Indeed, for BFS53 we determine a distance of $4.01 \pm 0.73 \mathrm{kpc}$ very different from the others.

On this basis we can split the complex into two complexes: complex 210.6-1.4 (BFS54 and S282) with a distance of $1.17 \pm 0.29 \mathrm{kpc}$ and BFS 53 (complex 211.14-1.01) placed at $4.01 \pm 0.73 \mathrm{kpc}$.

The complex 213.0-1.0: This complex is composed of the following HII regions: S283, S284, S285 and S286. For S286 no distance is available. Turbide \& Moffat (1993) obtained CCD $U B V$ magnitudes of stars towards S283, S284 and S285. Based on isochrones fits (assuming solar abundance) they determine distance of 7.3, 5.6 and $6.9 \mathrm{kpc}$ respectively for S283, S284 and S285.

We determine for $\mathrm{S} 283$ a distance a $8.06 \pm 0.30 \mathrm{kpc}$ from our stars 2 and 5. We determine distance for S284 and S285 from the photometric and spectroscopic results of Moffat et al. (1979) and Turbide \& Moffat (1993). A distance of $6.03 \pm 1.16 \mathrm{kpc}$ is deduced for S284 from star 1, 9 and 12 of Moffat et al. (1979) and a distance of $7.69 \pm 0.68 \mathrm{kpc}$ is deduced for $\mathrm{S} 285$ from star 1 and 6 of Moffat et al. (1979).

We will favour these last distance estimates for homogeneity. The distance for the complex is then $7.89 \pm 0.27 \mathrm{kpc}$.

The complex 224.2+1.2: The distance of this complex is the distance of S294. We find a new distance of $3.24 \pm 0.56 \mathrm{kpc}$. The old distance $(5.4 \mathrm{kpc})$ was determined from the same photometric data (Moffat et al. 1979) but with a photometric spectral type BOV.

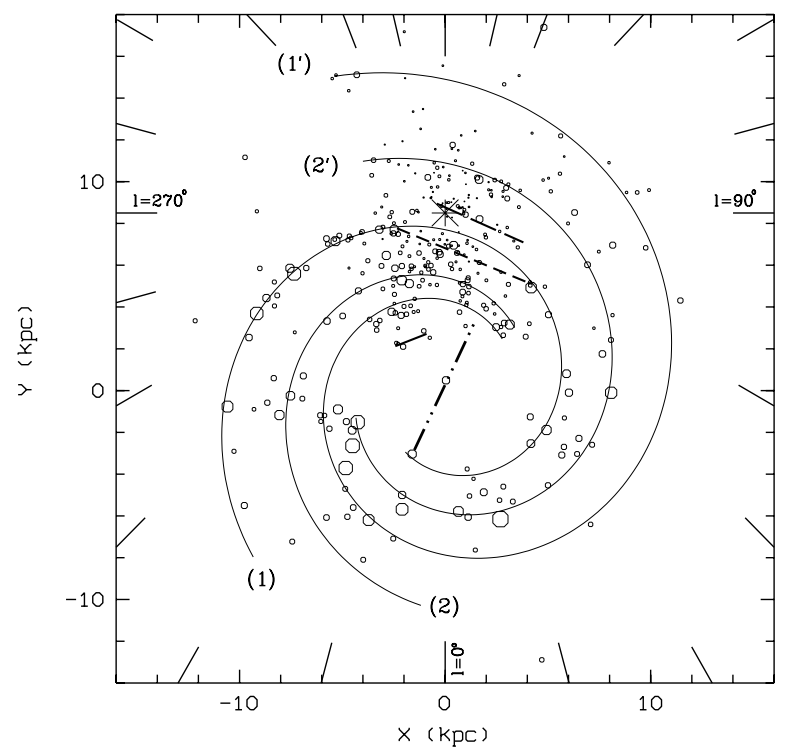

Fig. 4. Fitted spiral structure. The symbol size is proportional to the excitation parameter (see Russeil 2003). The Sun position is given by the large star symbol. 1: Sagittarius-Carina arm, 2: Scutum-Crux arm, $1^{\prime}$ : Norma-Cygnus arm and 2': Perseus arm. The local arm feature (long dashed line), the bar (dashed-dot-dot line, from Englmaier \& Gerhard 1999), the expected departure from the logarithmic spiral observed for the Sagittarius-Carina arm (short dashed line) and a feature probably linked to the 3-kpc arm (solid line) are sketched.

\section{Kinematic implications}

We take advantage of the new distance determinations to reinvestigate the kinematic of our Galaxy. Indeed, in the Perseus arm, velocity departures have been observed for a long time (e.g. Miller 1968; Rickard 1968; Roberts 1972; Humphreys 1976; Brand \& Blitz 1993). In the present discussion we have adopted for the Sun a galactocentric distance of $8.5 \mathrm{kpc}$ and for the solar circular velocity a value of $220 \mathrm{~km} \mathrm{~s}^{-1}$.

For the following kinematic study of the Galaxy, we adopt the analytical expression of the Brand \& Blitz (1993) rotation curve. Indeed, refitting the rotation curve (Fig. 3), we still find a result similar to that of Brand \& Blitz (1993). Due to the fact that most objects in this paper are at galactocentric distances between 10 and $12 \mathrm{kpc}$, where there are already lots of objects tracing the rotation curve, the new distance determinations were expected to have negligible effect on this rotation curve.

To investigate the velocity departures of star-forming complexes in the Perseus and Cygnus arms we first identify the complexes location respectively to each of these arms. We then fit the four arm model (Russeil 2003) taking into account the new stellar distances (Fig. 4). As expected, the large scale structure is not modified by the few new distances. Plotting the arms and the complexes in a galactocentric distance versus longitude diagram (Fig. 5) we determine the complexes association to every arm assuming that a complex belongs to an arm if its position is in the arm or if its error bar reaches the arm. To quantify the velocity departures we calculate the velocity difference deduced from the measured radial velocity and the stellar distance (Fig. 6), respectively.

The Perseus arm exhibits mean Vlsr departures of $-14.9 \pm$ $8.9 \mathrm{~km} \mathrm{~s}^{-1}$ in the second Galactic quadrant and $+5.17 \pm$ $9.14 \mathrm{~km} \mathrm{~s}^{-1}$ in the third Galactic quadrant. The Cygnus arm exhibits mean Vlsr departures $+8.06 \pm 9.95 \mathrm{~km} \mathrm{~s}^{-1}$ in the second Galactic quadrant and $-8.34 \pm 6.95 \mathrm{~km} \mathrm{~s}^{-1}$ in the third. 


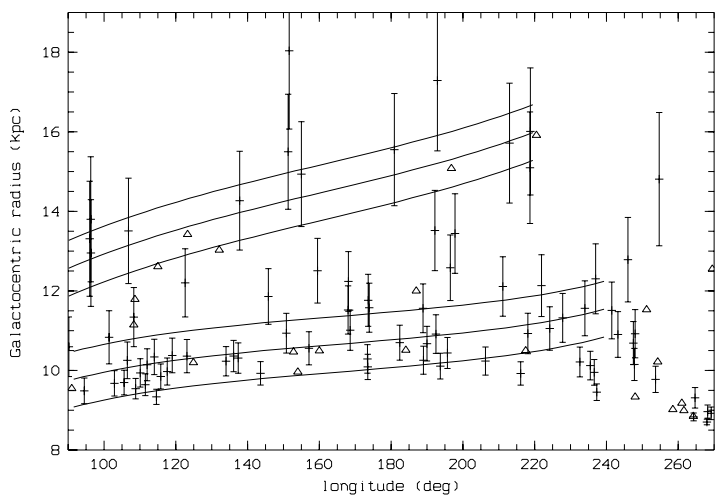

Fig. 5. Position of the complexes respectively to the fitted arms. Every arm is represented by three curves (central position and and width, $\pm 0.7 \mathrm{kpc}$, extension of the arm). Up is the cygnus arm, down is the Perseus arm. The triangle symbols are the complexes with kinematic distance only.

These values confirm our previous results (Russeil 2003) and are in agreement with the typical values observed in external galaxies. In addition, these velocity departures imply that the kinematic distance of the Perseus complexes are over-estimated while the kinematic distance to the Cygnus complexes are underestimated.

In external galaxies such motions are observed on large parts of arms (e.g. in NGC 300, Marcelin et al. 1985; in M 31, Ryden \& Stark 1986; in M 81, Adler \& Westpfahl 1996; in M 83, Tilanus \& Allen 1993; in NGC 1530, Zurita et al. 2004) from atomic, molecular and ionized gas velocity data. The amplitudes of these motions are typically between 10 and $40 \mathrm{~km} \mathrm{~s}^{-1}$.

In addition, we note that the velocity departures we obtain have opposite sign for the two arms. From HI data Burton (1973) and Burton \& Bania (1974) previously found negative velocity difference (for the second quadrant) at the location of the Perseus arm and positive difference at the location of the external arm (see e.g. Fig. 7 of Burton 1973). He showed that along a given line of sight the streaming motion has a periodic variation. Such a behaviour was modeled by Feitzinger \& Spicker (1987) from models of the radial velocity field of our Galaxy taking into account streaming motions due to spiral density wave.

Then, the velocity departures we obtain and their signs are well explained by the streaming motion along the arms. According to the density-wave theory, the perturbations of the gravitational potential in a rotating galaxy and the resulting spiral shocks give rise to streaming motion of young stars and gas (Lin et al. 1969; Roberts 1969). These streaming motions produce radial and azimuthal residual velocities. Sitnik et al. (2001) showed that inside the co-rotation radius the residual velocities should be maximum near the inner edge of the arm, where the shock front occurs. The radial and azimuthal residual velocities are directed toward the Galactic center and opposite to the Galactic rotation respectively. The magnitude of the residual velocity decreases with the distance to the edge of the arm. At the outer edge of the arm the radial residual velocity is close to zero and the azimuthal residual velocity is in the direction of the Galactic rotation. Outside the co-rotation radius the radial component of the streaming motion is directed away from the Galactic center and the azimuthal component is in the direction of the Galactic rotation (Mel'nik et al. 2001).

In this framework we can interpret the opposite sign of the velocity departures as indicating that the Perseus arm lies inside the co-rotation radius while the Cygnus arm lies outside it. In

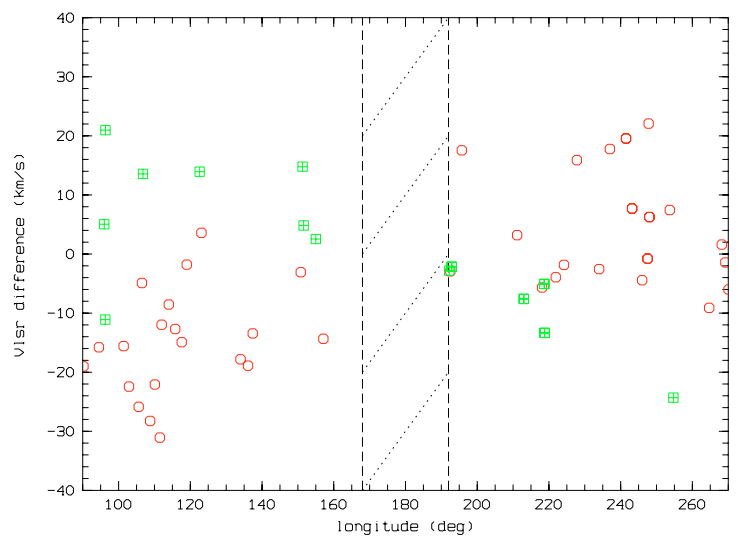

Fig. 6. Velocity difference versus longitude for the Perseus arm (circular symbol) and the Cygnus arm (square symbols).

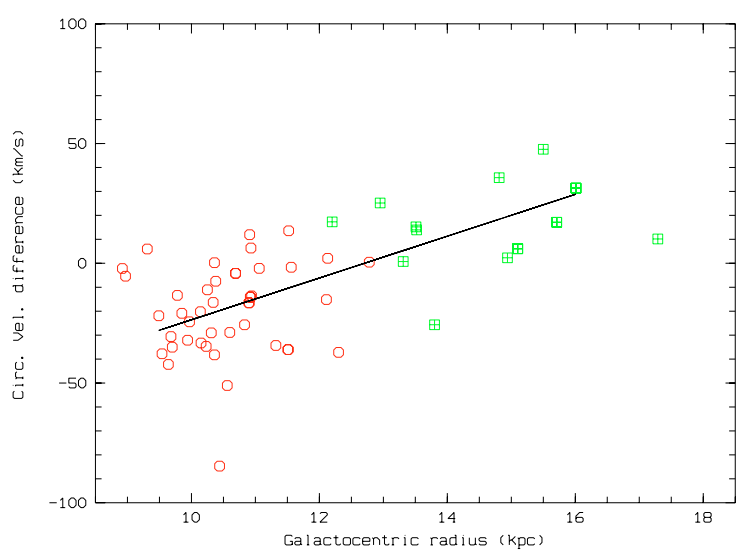

Fig. 7. Circular velocity difference versus galactocentric distance (the symbols are the same as in Fig. 6). The linear regression gives a slope of $8.74 \pm 1.10$ and a constant term of $-111.09 \pm 13.34$.

this way we find the co-rotation radius to be $12.7 \mathrm{kpc}$ (Fig. 7) from the fitted line expression.

This is higher than the usual value which places the corotation radius close to the Sun's location at a galactocentric distance $\sim 9 \mathrm{kpc}$. In the classical Lin et al. (1969) theory, the co-rotation resonance in our Galaxy is situated at the very end of the Galactic disc. However, Marochnik et al. (1972) and independently Crézé \& Mennessier (1973) came to the conclusion that the co-rotation is located close to the Solar position. This result has been supported by later work (e.g. Nelson \& Matsuda 1977; Amaral \& Lépine 1997; Mishurov et al. 1997; Mishurov \& Zenina 1999; Fernandez et al. 2001; Lépine et al. 2001; Luna et al. 2006; Dias et al. 2005). Recently, Popova \& Loktin (2005) found a co-rotation radius of about $10.5 \mathrm{kpc}$ from kinematic of open star clusters and OB stars.

\section{Conclusion}

Based on spectro-photometric observations we established or refined the stellar distance of HII regions, and then of their associated complex, of the outer Galaxy.

We reach the following main conclusions:

- The rotation curve is unchanged and still in agreement with the Brand \& Blitz (1993) rotation curve.

- We confirm the presence of departures from circular rotation in the Perseus arm and quantify such departures in the Cygnus arm. 
- These velocity departures are interpreted as streaming motions in the spiral arms. They suggest that the co-rotation radius of our Galaxy is located between the Perseus and Cygnus arms.

Acknowledgements. We thank the referee for useful comments and are grateful to the OHP team and to the students of the Aix-Marseille I RPA M2. We thank M.P. Ulmer for a careful reading of the paper and english corrections.

\section{References}

Adler, D. S., \& Westpfahl, D. J. 1996, AJ, 111, 735

Amaral, L. H., \& Lépine, J. R. D. 1997, MNRAS, 286, 885

Bertin, E., \& Arnouts, S. 1996, A\&A, 117, 393

Bica, E., Dutra, C. M., Soares, J., \& Barbuy, B. 2003, A\&A, 404, 223

Blitz, L., Fich, M., \& Stark, A. 1982, ApJS, 49, 183

Burton, W. B. 1973, PASP, 85, 679

Burton, W. B., \& Bania, T. M. 1974, A\&A, 33, 425

Bohigas, J., \& Tapia, M. 2003, AJ, 126, 1861

Bonnarel, F., Fernique, P., Bienaymé, O., et al. 2000, A\&AS, 143, 33

Brand, J., \& Blitz, L. 1993, A\&A, 275, 67

Brand, J., \& Wouterloot, J. 1994, A\&AS, 103, 503

Brunt, C., Kerton, C., \& Pomerleau, C. 2003, ApJS, 144, 47

Carrasco-Gonzalez, C., Lopez, R., Gyulbudaghian, A., et al. 2006, A\&A, 445, L43

Chini, R., \& Wink, J. E. 1984, A\&A, 139, L5

Cohen, M., \& Green, A. J. 2001, MNRAS, 325, 531

Conti, P. S., \& Underhill, A. B. 1988, O Stars and Wolf-Rayet Stars, NASA SP497

Crampton, D., Georgelin, Y. M., \& Georgelin, Y. P. 1978, A\&A, 66, 1

Crézé, M., \& Mennessier, M. O. 1973, A\&A, 27, 281

Dame, T., Ungerechts, H., Cohen, R., et al. 1987, ApJ, 322, 706

Deharveng, L., Zavagno, A., Nadeau, D., Caplan, J., \& Petit, M. 1999, A\&A, 344,943

Deharveng, L., Zavagno, A., Salas, L., et al. 2003, A\&A, 399, 1135

Dennison, B., Topasma, G., \& Simonetti, J. 1997, ApJ, 474, L3

Dias, W. S., \& Lépine, J. R. D. 2005, ApJ, 629, 825

Digel, S., de Geus, E., \& Thaddeus, P. 1994, ApJ, 422, 92

Digel, S., Lyder, D. A., Philbrick, A. J., et al. 1996, ApJ, 458, 561

Englmaier, P., \& Gerhard, O. 1999, MNRAS, 304, 512

Feitzinger, J. V., \& Spicker, J. 1987, A\&A, 184, 122

Felli, M., \& Perinotto, M. 1974, ApSS, 26, 115

Fernandez, D., Figueras, F., \& Torra, J. 2001, A\&A, 372, 833

Fich, M. 1993, ApJS, 86, 475

Fich, M., \& Blitz, L. 1984, ApJ, 279, 125

Fich, M., Dahl, G., \& Treffers, R. 1990, AJ, 99, 622

Foster, T., \& MacWilliams, J. 2006, ApJ, 644, 214

Georgelin, Y. M., Georgelin, Y. P., \& Roux, S. 1973, A\&A, 25, 337

de Geus, E., Vogel, S. N., Digel, S., \& Gruendl, R. A. 1993, ApJ, 413, L97

Harris, H. C., Dahn, C. C., Monet, D. G., \& Pier, J. R. 1997, in Planetary Nebulae, ed. H. J. Habing, \& L. M. Lamers (Dordrecht: Kluwer Academic Publ.), IAU Symp., 180, 40

Haug, U. 1970, A\&AS, 1,35

Heyer, M. H., \& Terebey, S. 1998, ApJ, 502, 265

Heyer, M. H., Brunt, C., Snell, R., et al. 1998, ApJS, 115, 241

Hiltner, W., \& Johnson, H. 1956, ApJ, 124, 367

Humphreys, R. M. 1970, AJ, 75, 602
Humphreys, R. M. 1976, ApJ, 206, 114

Hunter, D. A., \& Massey, P. 1990, AJ, 99, 846

Jaschek, C., \& Jaschek, M. 1987, The classification of stars (Cambridge University Press)

Kaltcheva, N. T., \& Hilditch, R. W. 2000, MNRAS, 312, 753

Karchenko, N. 2001, KFNT, 17, 409

Kelly, D. M., \& Hrivnak, B. J. 2005, ApJ, 629, 1040

Kerton, C. R., Brunt, C. M., \& Kothes, R. 2004, AJ, 127, 1059

Kimeswenger, S., \& Weinberger, R. 1989, A\&A, 209, 51

Kobayashi, N., \& Tokunaga, A. T. 2000, ApJ, 532, 423

Kutner, M., \& Mead, K. 1981, ApJ, 249, L15

Lahulla, J. F. 1987, AJ, 94, 1062

Landecker, T. L., Anderson, M. D., Routledge, D., \& Vaneldik, J. F. 1992, A\&A, 258,495

Landolt, A. U. 1983, AJ, 88, 853

Leger, A., \& Puget, J. L. 1984, A\&A, 137, L5

Lemaitre, G., Kohler, D., Lacroix, D., Meunier, J. P., \& Vin, A. 1990, A\&A, 228, 546

Lépine, J., Mishurov, Y., \& Dedikov, S. 2001, ApJ, 546, 234

Lin, C. C., Yuan, C., \& Shu, F. H. 1969, ApJ, 155, 721

Luna, A., Bronfman, L., Carrasco, L., \& May, J. 2006, ApJ, 641, 938

Marcelin, M., Boulesteix, J., \& Georgelin, Y. P. 1985, A\&A, 151, 144

Marochnik, L. S., Mishurov, Yu., N., \& Suchkov, A. A. 1972, Ap\&SS, 19, 285

May, J., Alvarez, H., \& Bronfman, L. 1997, A\&A, 327, 325

Mel'Nik, A. M., Dambis, A. K., \& Rastorguev, A. S. 2001, AstL, 27, 521

Meyer, S. 1975, in Data analysis for scientists and engineers (Wiley Series)

Miller, J. S. 1968, ApJ, 151, 473

Mirzoyan, L., Boulesteix, J., Hambarian, V., et al. 1994, Astrophys., 37, 105

Mishurov, Yu. N., Zenina, I. A., Dambis, A. K., et al. 1997, A\&A, 323, 775

Mishurov, Yu. N., \& Zenina, I. A. 1999, A\&A, 341, 81

Moffat, A., Jackson, P., \& Fitzgerald, M. P. 1979, A\&AS, 38, 197

Muzzio, J. C., \& Rydgren, A. E. 1974, AJ, 79, 864

Nakagawa, M., Onoshi, T., Mizuno, A., et al. 2005, PASJ, 57, 917

Napiwotzki, R. 2001, A\&A, 367, 973

Negueruela, I., \& Marco, A. 2003, A\&A, 406, 119

Nelson, A. H., \& Matsuda, T. 1977, MNRAS, 179, 663

Nesterov, V., Kuzmin, A., Ashimbaeva, N., et al. 1995, A\&AS, 110, 367

Nicolet, B. 1978, A\&AS, 34, 1

Pismis, P. 1990, A\&A, 234, 443

Pismis, P., \& Hasse, I. 1980, RMxAA, 5, 39

Price, S. D., Egan, M. P., Carey, S. J., Mizuno, D. R., \& Kuchar, T. A. 2001, AJ, 121,2819

Popova, M. E., \& Loktin, A. V. 2005, AstL, 31, 663

Reed, C. 1998, ApJS, 115, 271

Rickard, J. J. 1968, ApJ, 152, 1019

Roberts, W. W. 1969, ApJ, 158, 23

Roberts, W. 1972, ApJ, 173, 259

Russeil, D. 2003, A\&A, 397, 133

Ryden, B. S., \& Stark, A. A. 1986, ApJ, 305, 823

Sitnik, T. G., Mel'Nik, A. M., \& Pravdikova, V. V. 2001, ARep, 45, 34

Smartt, S. J., Dufton, P. L., \& Rolleston, W. R. J. 1996, A\&A, 310, 123

Tilanus, R. P., \& Allen, R. J. 1993, A\&A, 274, 707

Turbide, L., \& Moffat, A. 1993, AJ, 105, 1831

Vallée, J. P. 2005, AJ, 130, 569

Walborn, N. R., \& Fitzpatrick, E. L. 1990, PASP, 102, 379

Wegner, W. 1994, MNRAS, 270, 229

Wouterloot, J., \& Brand, J. 1989, A\&AS, 80, 149

Wramdemark, S. 1981, A\&AS, 43, 103

Zurita, A., Relano, M., Beckman, J. E., \& Knapen, J. H. 2004, A\&A, 413, 73 
D. Russeil et al.: Revised distances of Northern HII regions, Online Material p 1

\section{Online Material}


D. Russeil et al.: Revised distances of Northern HII regions, Online Material p 2

\section{Appendix A: Identification charts and spectra}

For every HII region we present the identification chart of the exciting stars (Figs. A.1 to A.19). The stars are indicated by a short line labeled (when necessary) by the star identification. Overlaid on the images are MSX Band A contours or $4.89 \mathrm{GHz}$ radio contours (GB6 sky survey). For every image the north is up and east is left. The spectra of every exciting star is also presented (Figs. A.20 to A.23). The spectra of the late-type stars are available on request. The Tables A.1 and A.2 summarise respectively the galactocentric distance and the composition of the complexes and the distance of the individual HII regions.

Table A.1. Galactocentric distance of the star-forming complexes.

\begin{tabular}{lccc}
\hline \hline Complex & HII regions & $\begin{array}{c}\text { Old } \\
\text { distance } \\
\mathrm{kpc}\end{array}$ & $\begin{array}{c}\text { New } \\
\text { distance } \\
\mathrm{kpc}\end{array}$ \\
\hline $96.00+2.00$ & BFS6,BFS7,S128 & $12.1 \pm 1.6$ & $13.3 \pm 0.3$ \\
$96.30-0.20$ & BFS8 & - & $12.9 \pm 0.5$ \\
$101.40+2.70$ & BFS10,DA568 & - & $10.8 \pm 0.2$ \\
$105.60+0.40$ & S138,S139 & $10.3 \pm 0.7$ & $9.7 \pm 0.7$ \\
$106.80+3.30$ & S141 & - & $13.5 \pm 0.5$ \\
$108.80-1.00$ & S152,S153 & $10.3 \pm 0.5$ & $9.5 \pm 0.1$ \\
$110.10+0.00$ & BFS14,BFS15,BFS16 & $10.7 \pm 0.6$ & $9.9 \pm 0.4$ \\
& BFS17,BFS18,S156 & & \\
$111.50-0.80$ & S158,S159 & $10.7 \pm 0.6$ & $9.6 \pm 0.1$ \\
$114.00-0.70$ & S163,S164,S166 & $9.7 \pm 0.3$ & $10.4 \pm 0.1$ \\
$117.60+2.30$ & S170 & $10.1 \pm 0.4$ & $9.9 \pm 0.2$ \\
$119.00-1.10$ & S172,S173,S177 & $9.9 \pm 0.4$ & $10.4 \pm 0.2$ \\
$120.40+2.00$ & S175 & $9.9 \pm 0.5$ & $9.1 \pm 0.1$ \\
$126.70-1.00$ & S187 & - & $9.4 \pm 0.2$ \\
$136.10+2.10$ & S192,S193,S196 & - & $10.4 \pm 0.3$ \\
$137.80-1.00$ & & - & $14.3 \pm 0.5$ \\
$143.60-1.80$ & S203,BFS31 & - & $9.9 \pm 0.3$ \\
$145.80+3.00$ & S204 & $12.0 \pm 1.1$ & $11.9 \pm 0.2$ \\
$159.50+2.50$ & S219,S217,BFS44 & $14.4 \pm 0.7$ & $12.5 \pm 0.3$ \\
$192.50-0.10$ & S254,S255,S256 & $10.8 \pm 0.3$ & $10.9 \pm 0.2$ \\
\multicolumn{4}{c}{ S257,S258 } \\
$210.60-1.40$ & BFS54,S282 & $10.2 \pm 0.4$ & $9.5 \pm 0.3$ \\
$211.14-1.01$ & BFS53 & - & $12.1 \pm 0.7$ \\
$213.00-1.00$ & S283,S284,S285 & $14.5 \pm 0.3$ & $15.7 \pm 0.3$ \\
& S286 & & \\
$224.20+1.20$ & S294 & $12.9 \pm 1.4$ & $11.1 \pm 0.5$ \\
\hline & & &
\end{tabular}

Table A.2. Revised stellar distances of the individual HII regions.

\begin{tabular}{lc}
\hline \hline HII region & $\begin{array}{c}\text { Distance } \\
\mathrm{kpc}\end{array}$ \\
\hline BFS8 & $8.8 \pm 0.61$ \\
DA568 & $4.99 \pm 0.32$ \\
BFS10 & $6.39 \pm 0.67$ \\
S138 & $2.91 \pm 1.2$ \\
S141 & $8.34 \pm 0.6$ \\
S152 & $2.39 \pm 0.21$ \\
S153 & $4.73 \pm 0.6$ \\
S156 & $2.87 \pm 0.75$ \\
S158 & $2.27 \pm 0.15$ \\
S159 & $2.97 \pm 0.31$ \\
S163 & $4 \pm 0.22$ \\
S170 & $2.39 \pm 0.17$ \\
S173 & $3.12 \pm 0.34$ \\
S175 & $1.09 \pm 0.21$ \\
S187 & $1.44 \pm 0.26$ \\
S192 & $2.96 \pm 0.54$ \\
S193 & $2.11 \pm 0.39$ \\
G137.8+1.0 & $6.78 \pm 0.59$ \\
S196 & $5.67 \pm 0.59$ \\
BFS31 & $1.71 \pm 0.31$ \\
S204 & $3.83 \pm 0.42$ \\
S217 & $4.37 \pm 0.37$ \\
S219 & $3.99 \pm 0.39$ \\
S256 & $2.14 \pm 0.39$ \\
S258 & $2.9 \pm 0.51$ \\
S283 & $8.06 \pm 0.30$ \\
S294 & $3.24 \pm 0.56$ \\
BFS53 & $4.01 \pm 0.73$ \\
\hline & \\
&
\end{tabular}


D. Russeil et al.: Revised distances of Northern HII regions, Online Material p 3
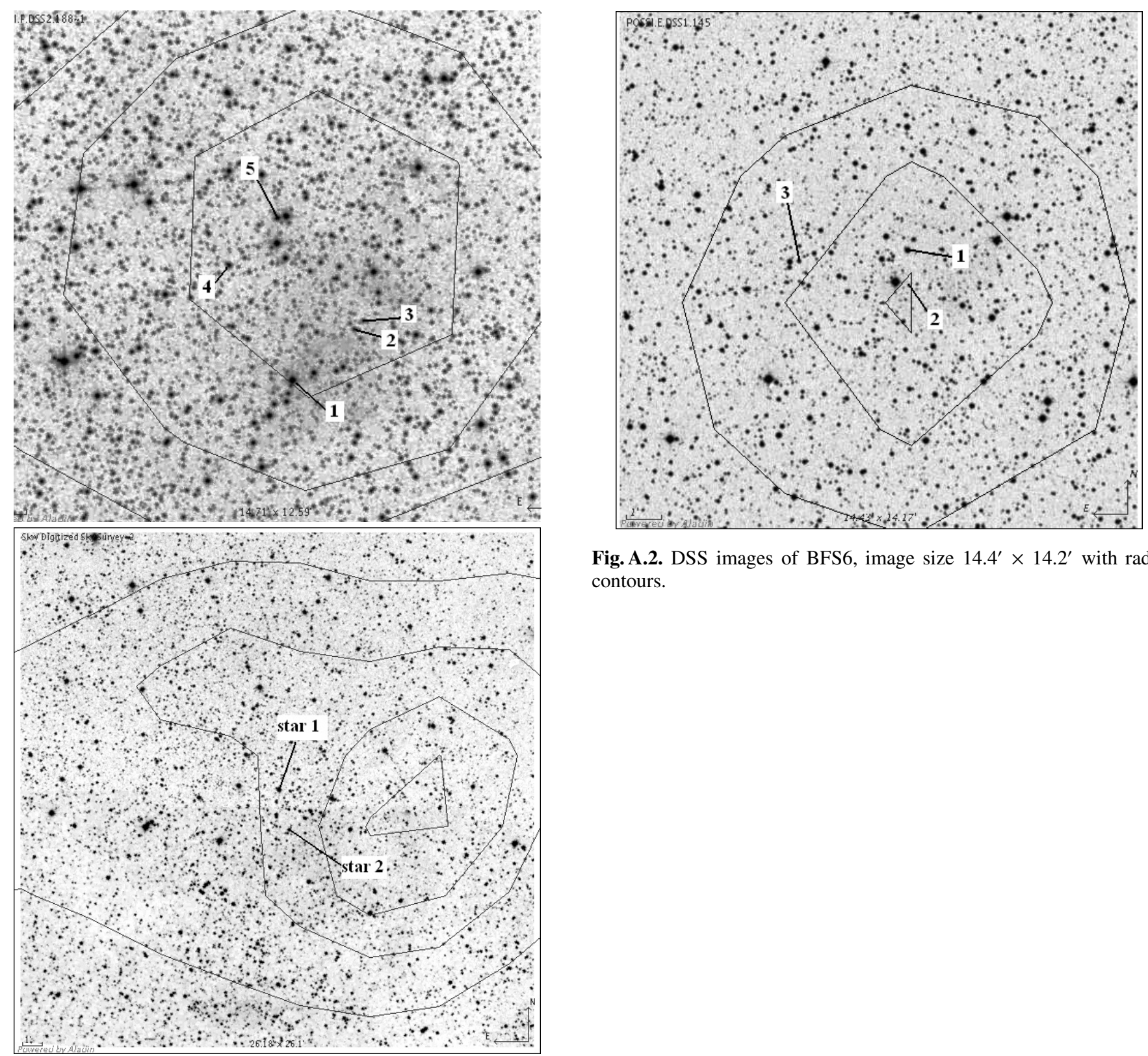

Fig. A.2. DSS images of BFS6, image size $14.4^{\prime} \times 14.2^{\prime}$ with radio contours.

Fig. A.1. DSS images of BFS8 (top), image size $14.7^{\prime} \times 12.6^{\prime}$ and DA568 (bottom) with radio contours, image size $26.2^{\prime} \times 26.1^{\prime}$. 
D. Russeil et al.: Revised distances of Northern HII regions, Online Material p 4
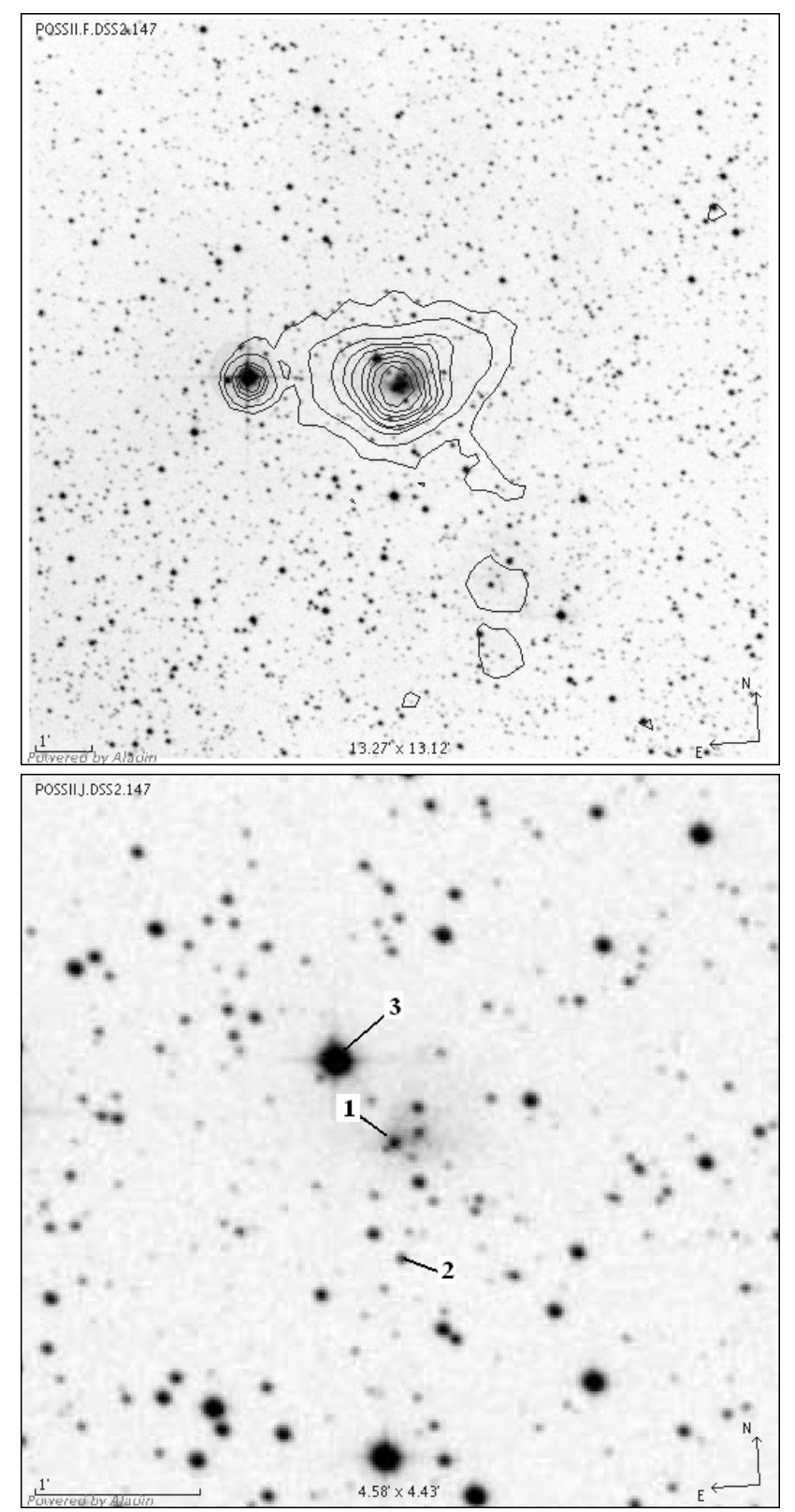

Fig. A.3. DSS images of S138. Top: red image and MSX Band A contours (image size $13 \cdot 3^{\prime} \times 13 \cdot 1^{\prime}$ ). Bottom: blue image which allows us to better identify the star (image size $4.6^{\prime} \times 4.4^{\prime}$ ).

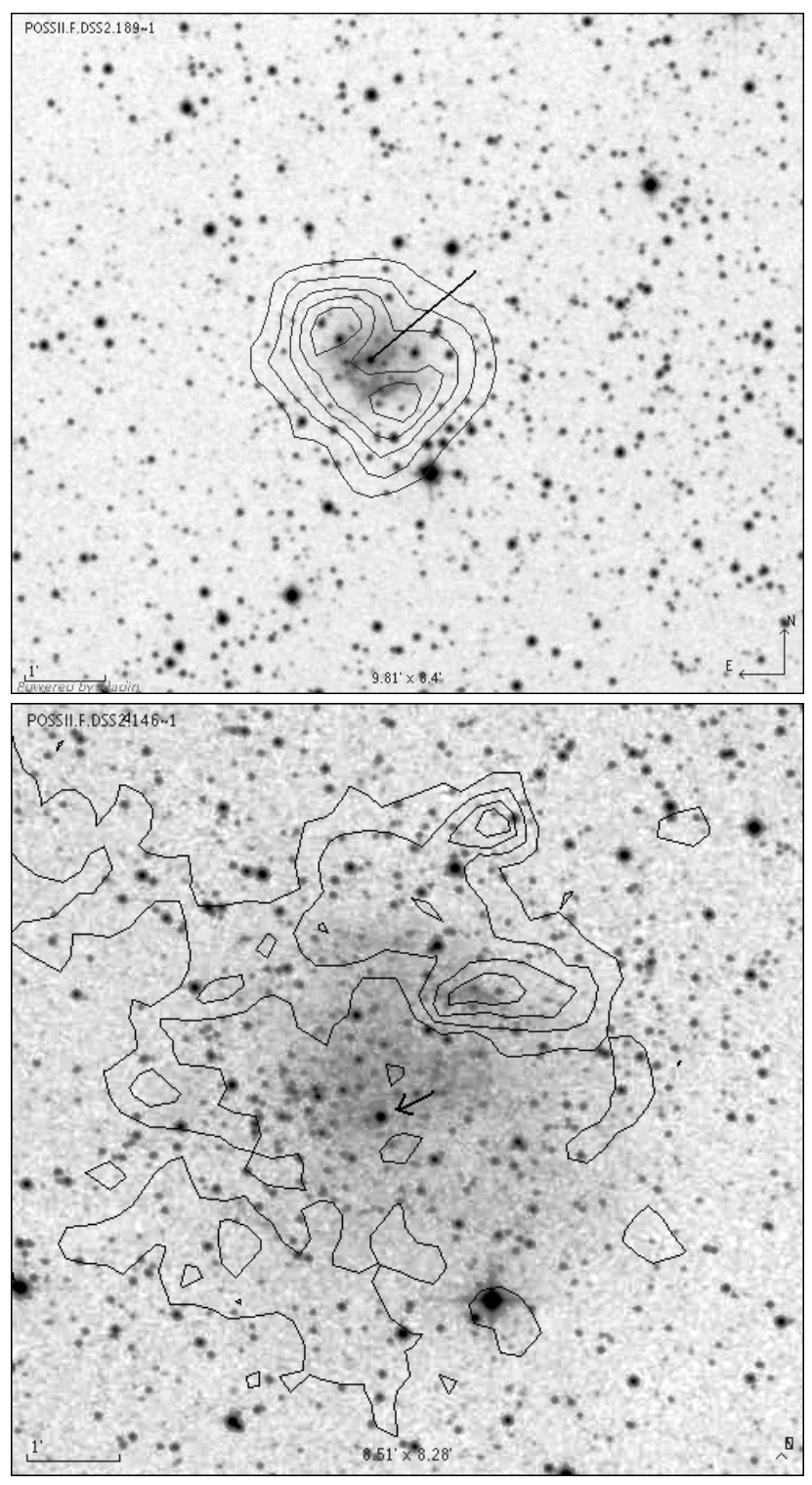

Fig. A.4. DSS images of BFS10 (top), image size $9.8^{\prime} \times 8.4^{\prime}$ and S141 (bottom) with overlaid MSX Band A contours, image size $8.5^{\prime} \times 8.3^{\prime}$. 
D. Russeil et al.: Revised distances of Northern HII regions, Online Material p 5

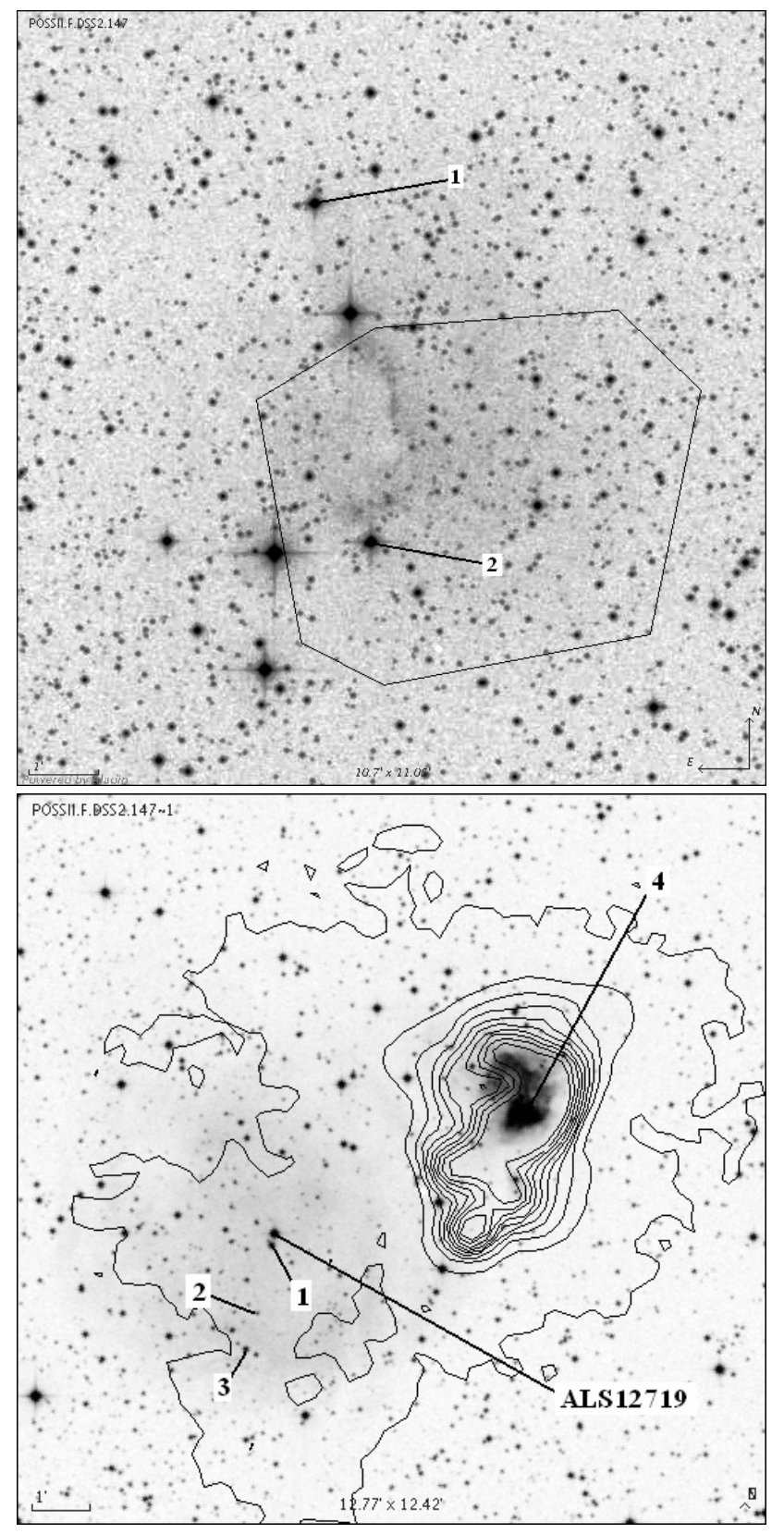

Fig. A.5. Top: DSS image with radio contours of S144 (top), image size $11^{\prime} \times 11^{\prime}$. Botton: DSS image with MSX Band A contours of S152/S153, image size $12.8^{\prime} \times 12.4^{\prime}$

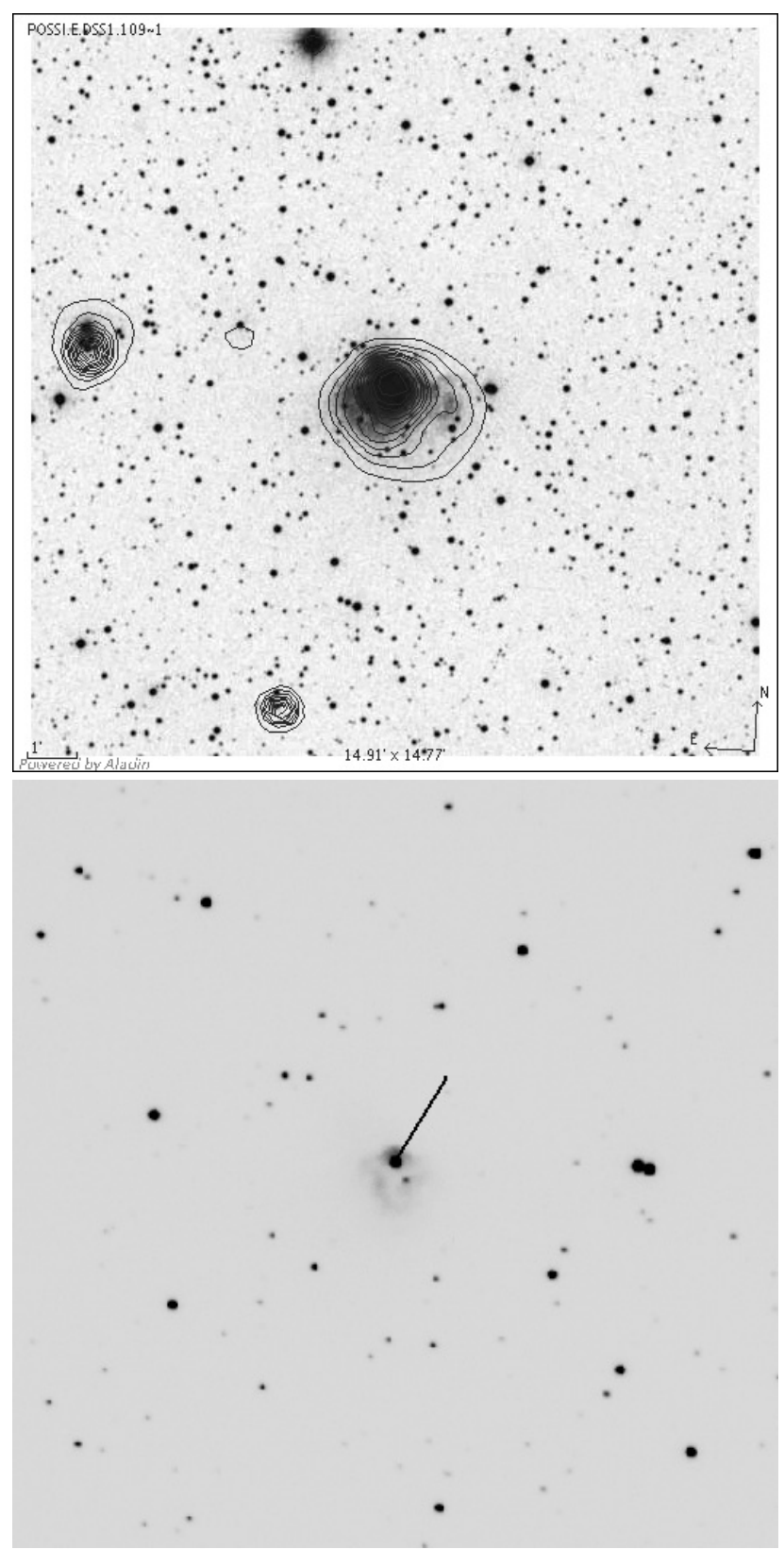

Fig. A.6. Top: DSS image of S156 with MSX Band A contours $\left(14.9^{\prime} \times\right.$ $14.8^{\prime}$ size $)$. Bottom: our $V$-band image $\left(6^{\prime} \times 6^{\prime}\right.$ size $)$. 
D. Russeil et al.: Revised distances of Northern HII regions, Online Material p 6

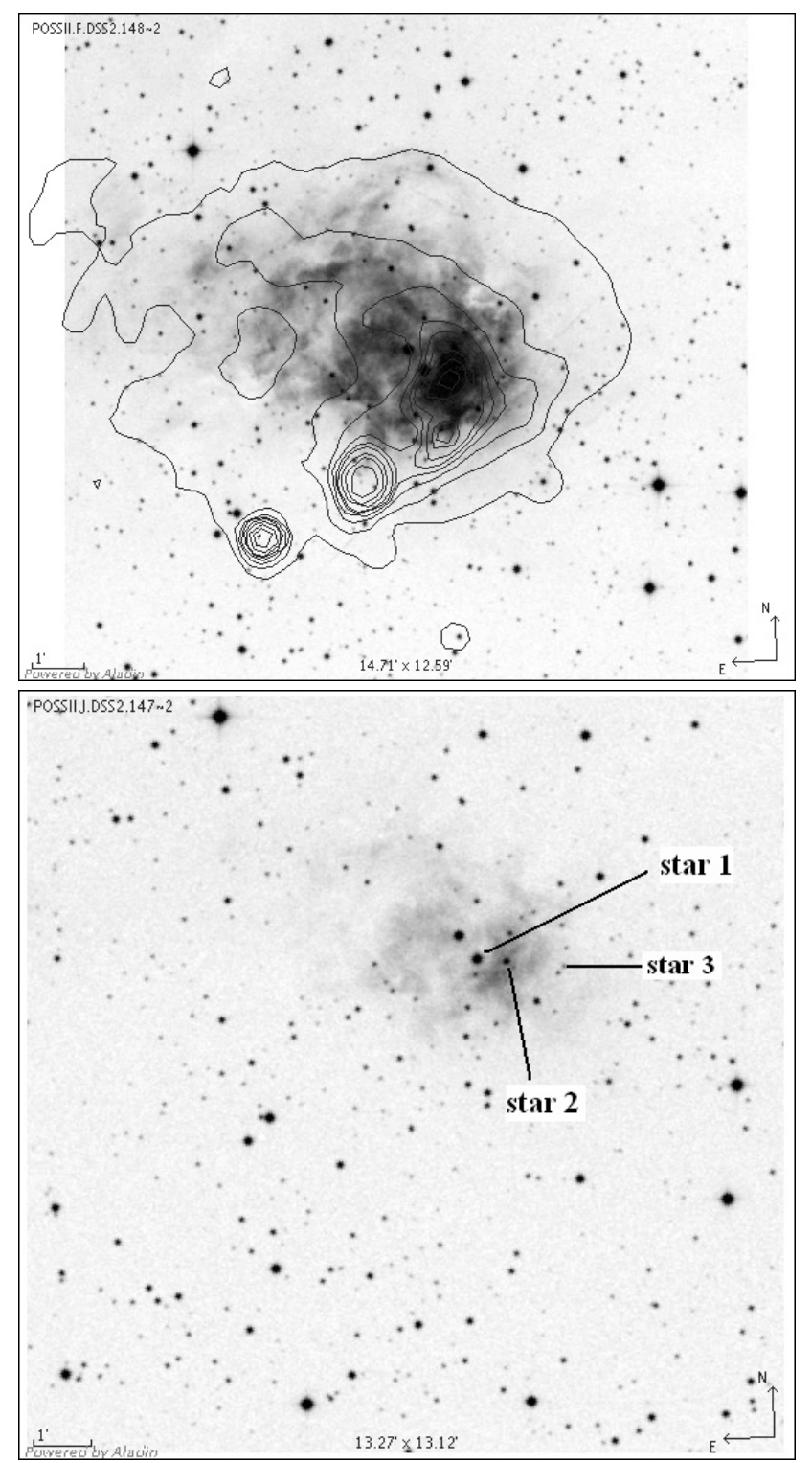

Fig. A.7. DSS images of S158. Top: red image and MSX band A contours $\left(14.7^{\prime} \times 12.6^{\prime}\right.$ size $)$. Bottom: blue image with star identification $\left(13.3^{\prime} \times 13.1^{\prime}\right.$ size $)$.

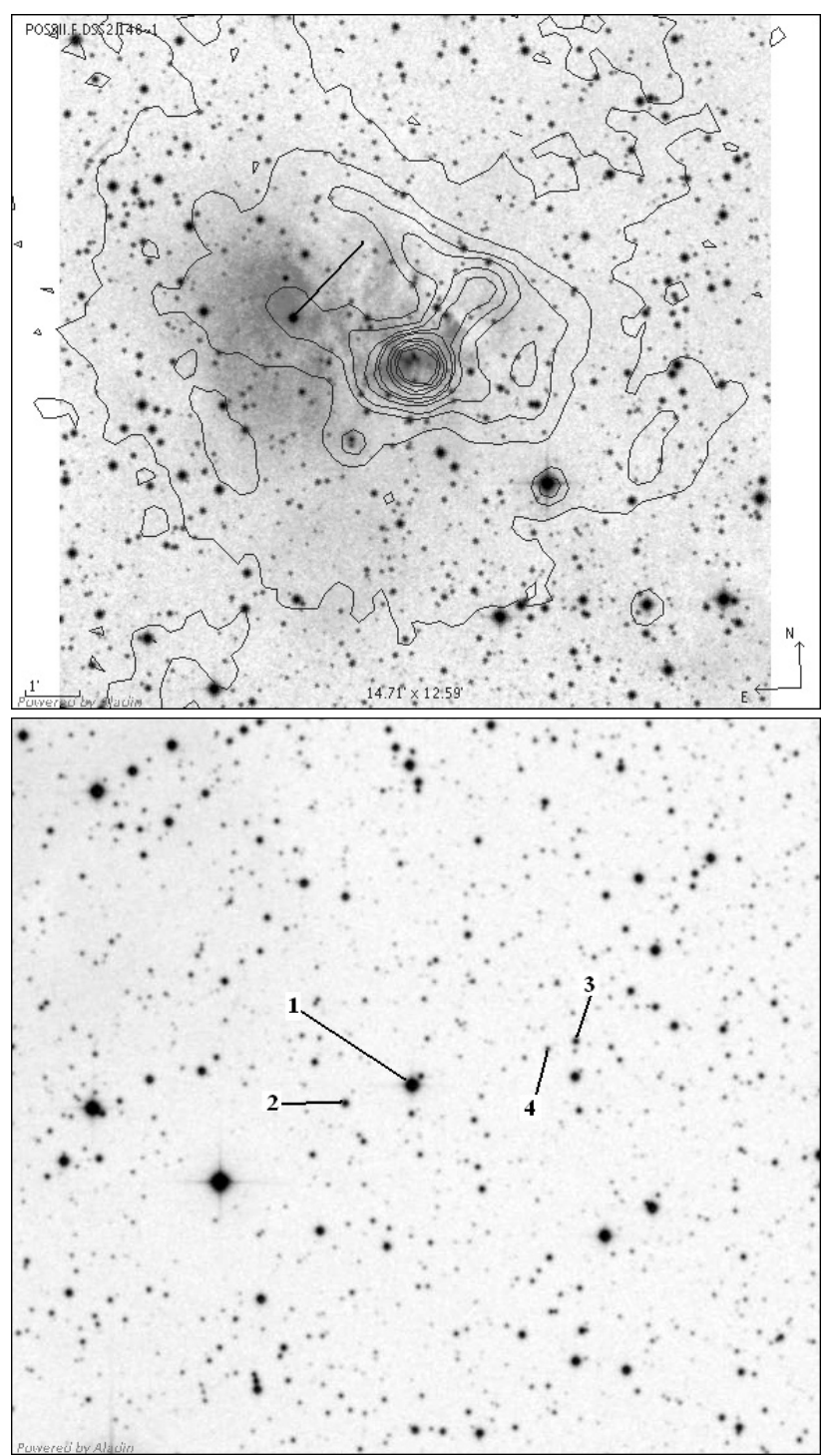

Fig. A.8. DSS images with MSX band A contours of s159 (top), image size $14.7^{\prime} \times 12.6^{\prime}$ and $\mathrm{S} 163$ stars 1 to 4 (bottom), image size $10.3^{\prime} \times 9.8^{\prime}$. 
D. Russeil et al.: Revised distances of Northern HII regions, Online Material p 7

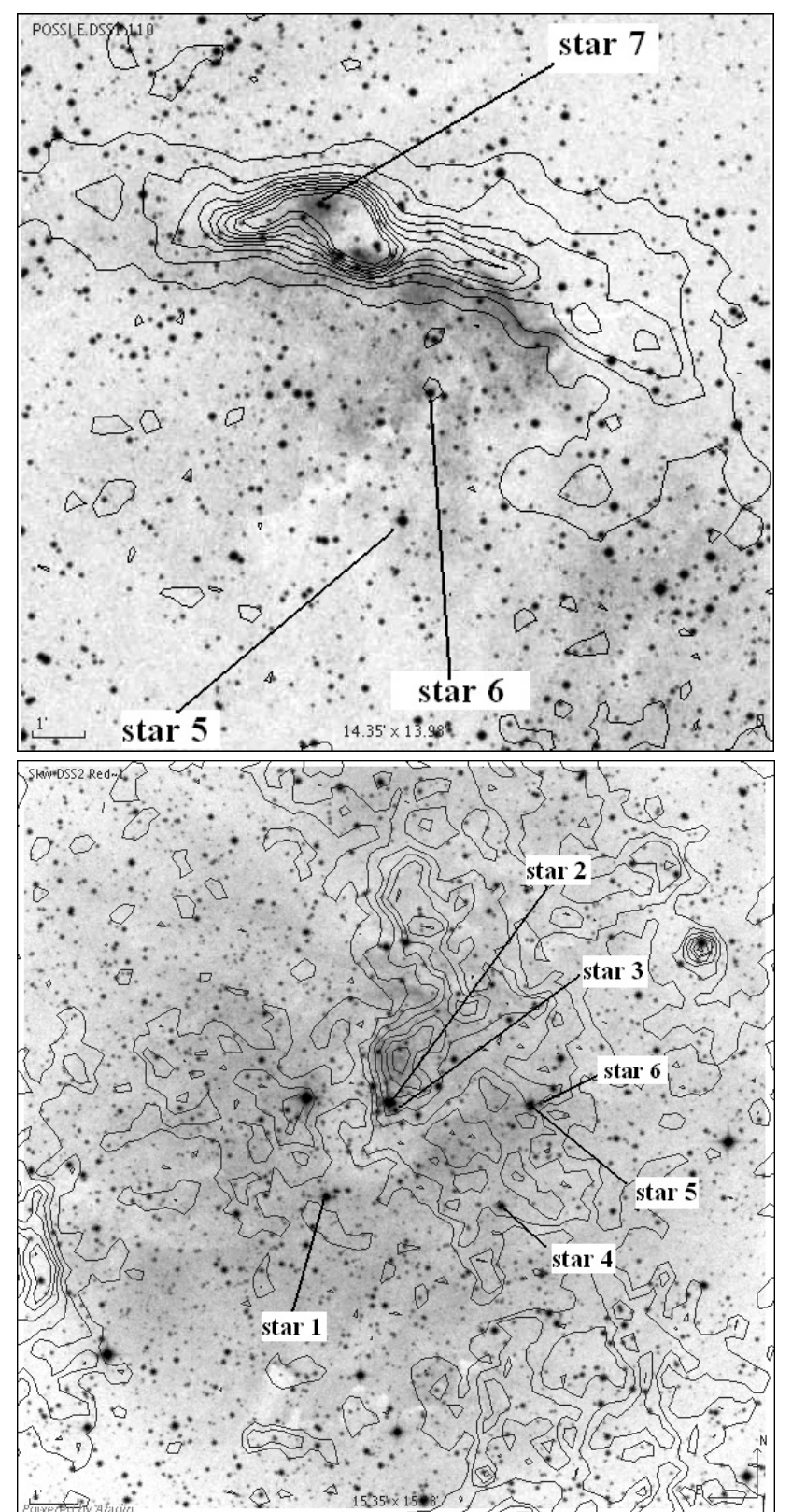

Fig. A.9. DSS images with $M S X$ band A contours of S163 (top), image size $14.3^{\prime} \times 14^{\prime}$ and S170 (bottom), image size $15.3^{\prime} \times 15.3^{\prime}$.

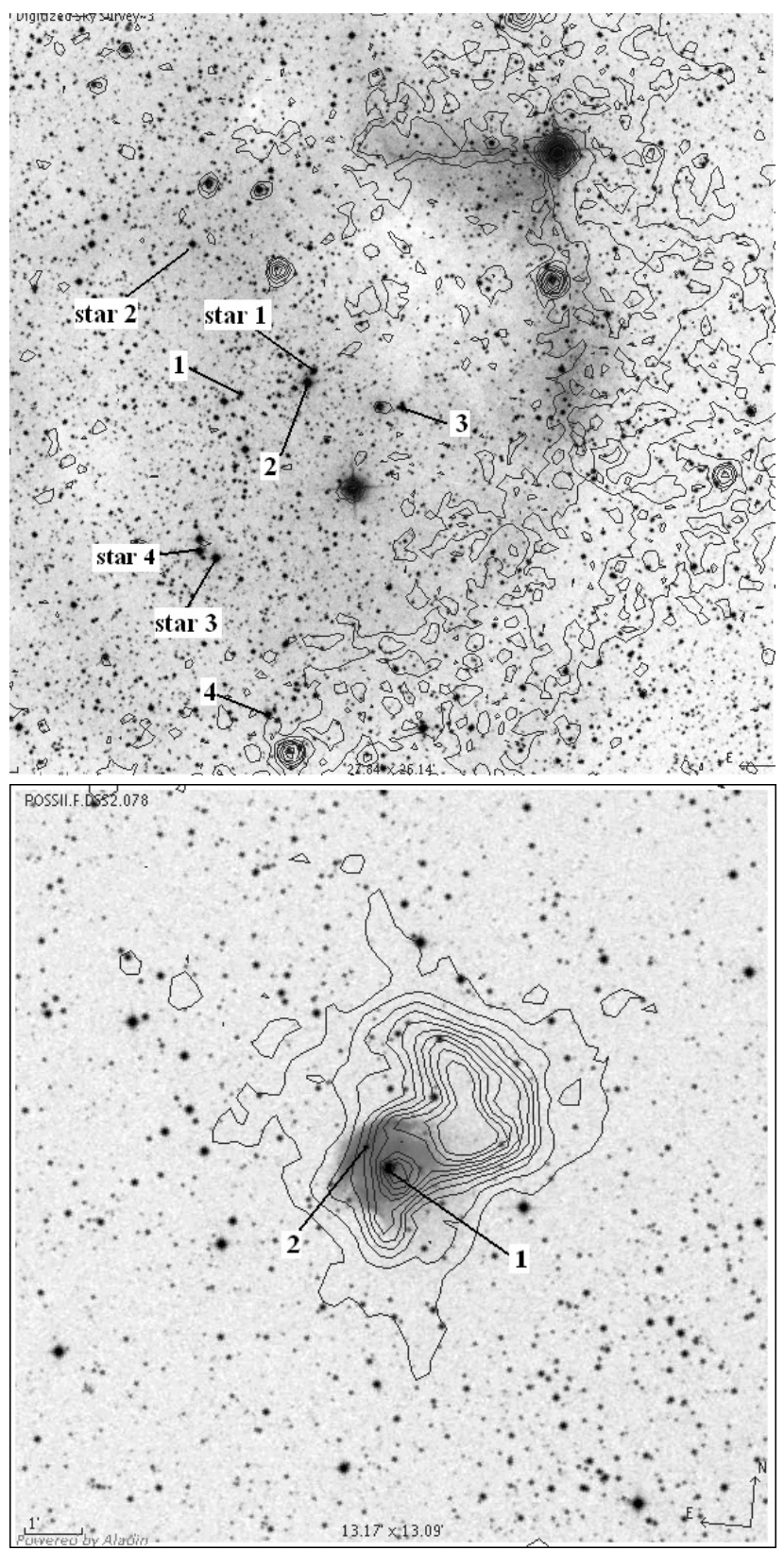

Fig. A.10. DSS image with $M S X$ band A contours of S173 (top), image size $27.8^{\prime} \times 25.1^{\prime}$. The labels "star 1 " to "star 4" refer to the stars observed in this paper. The labels "1" to "4" refer to the stars from the literature discussed in the text: 1) ALS6156, 2) ALS6151, 3) ALS6145 and 4) ALS6155. Bottom: DSS image with MSX band A contours of S175, image size $13.2^{\prime} \times 13.1^{\prime}$. 
D. Russeil et al.: Revised distances of Northern HII regions, Online Material $p 8$

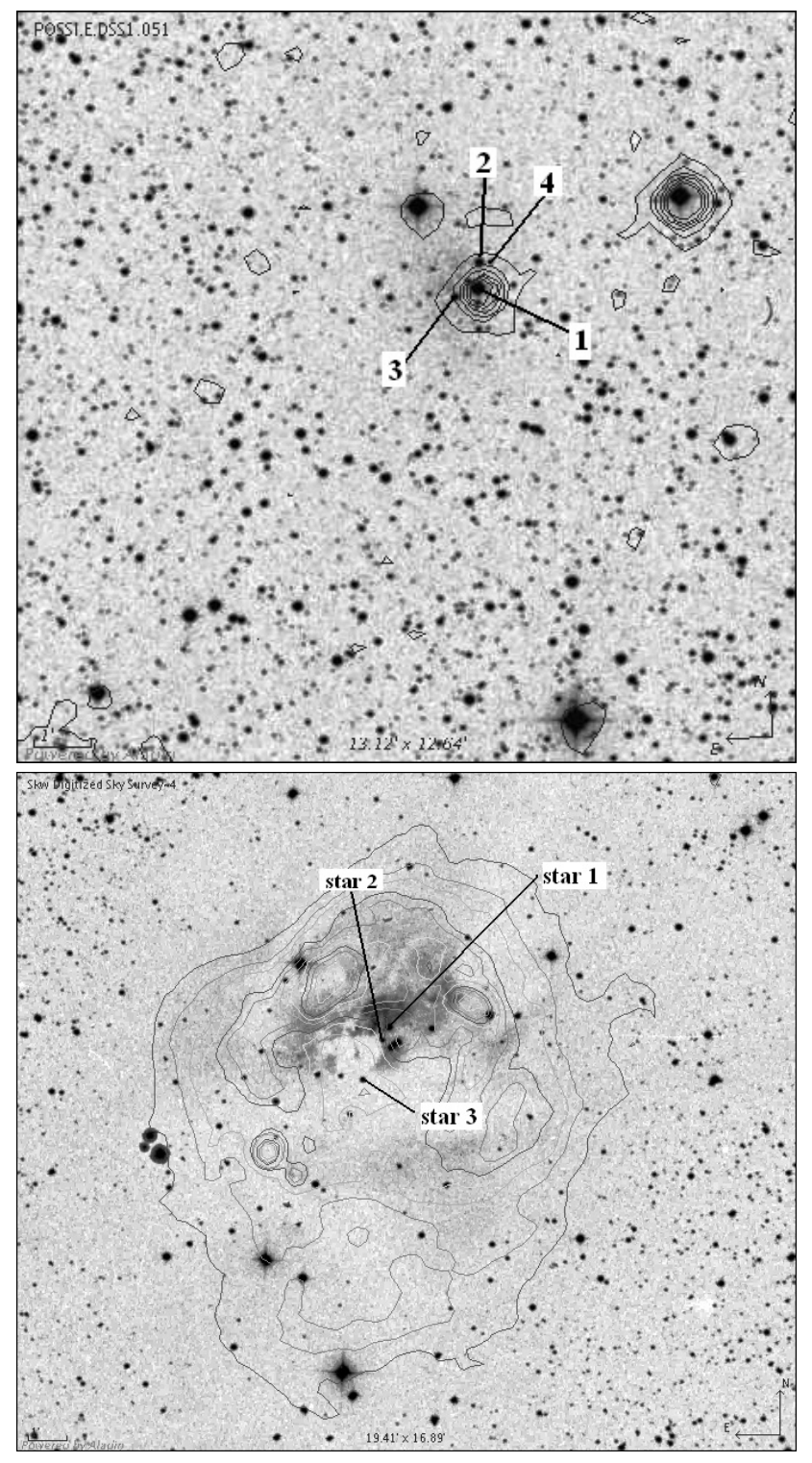

Fig. A.11. DSS images with MSX band A contours of S182 (top), image size $13.1^{\prime} \times 12.6^{\prime}$ and S187 (bottom), image size $19.4^{\prime} \times 16.9^{\prime}$.

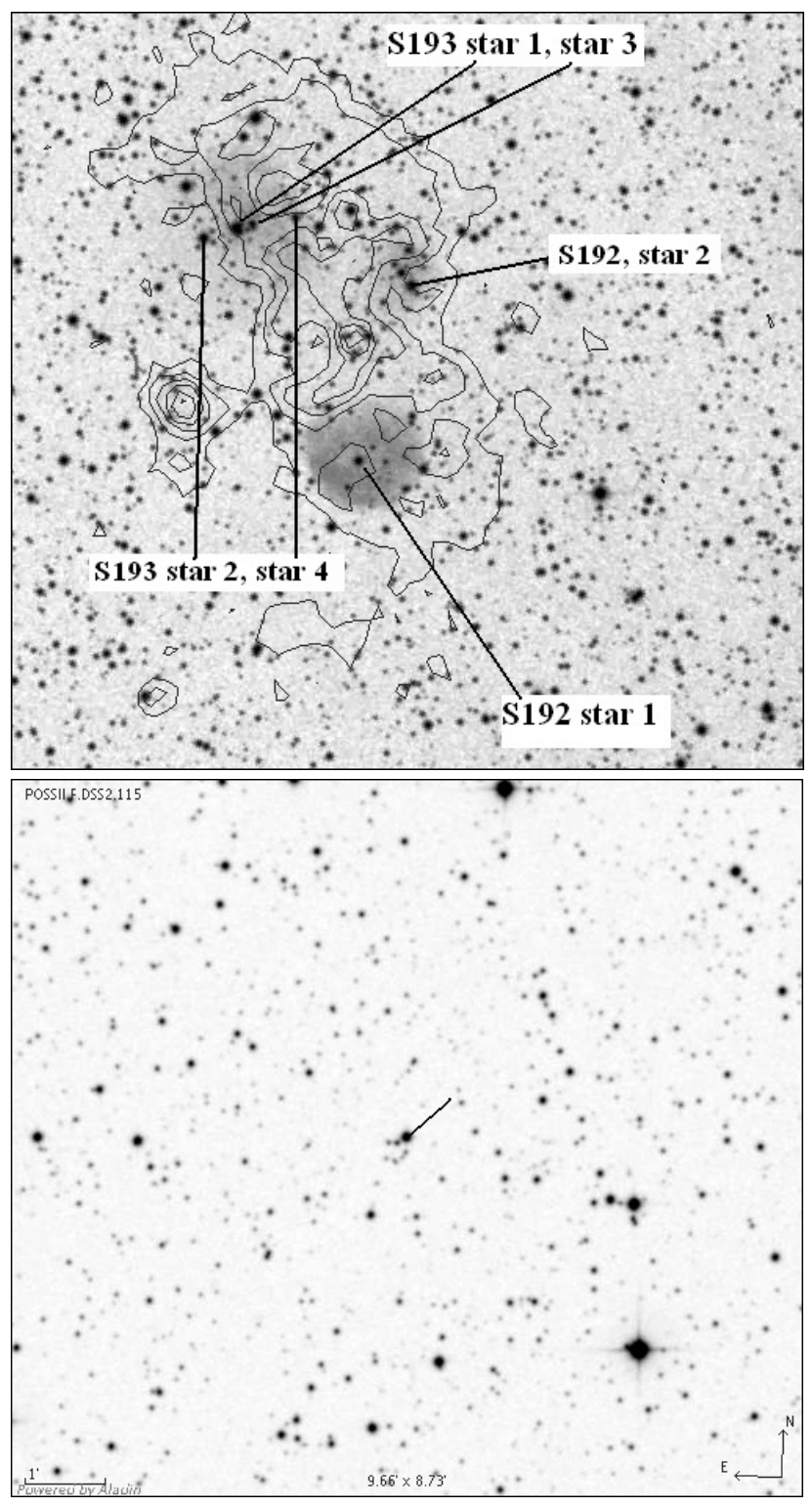

Fig. A.12. DSS image with MSX band A contours of S192 and S193 (top), image size $12.8^{\prime} \times 12.1^{\prime}$. Bottom: DSS image of the exciting star of G137.8-1.0, image size $9.7^{\prime} \times 8.7^{\prime}$. 
D. Russeil et al.: Revised distances of Northern HII regions, Online Material p 9
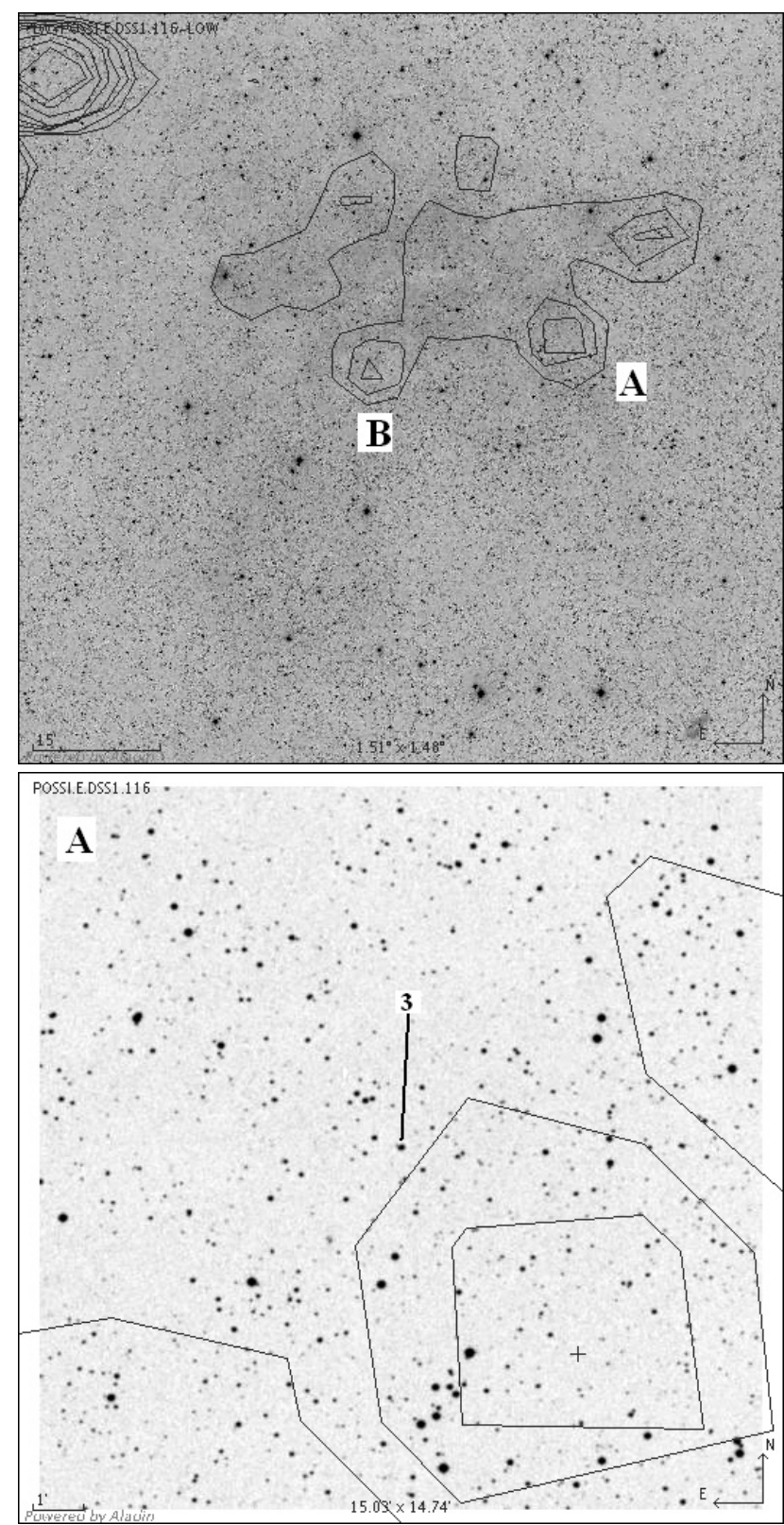

Fig. A.13. Top: DSS image of S203 with radio contours (image size $1.5^{\circ} \times 1.5^{\circ}$ ). For the two locations noted $\mathrm{A}$ and $\mathrm{B}$ we give detailed images. Bottom: smaller scale image (location A) with the identified exciting star (image size $15^{\prime} \times 14.7^{\prime}$ ). The cross indicates the radio source GB6 B0316+5439.

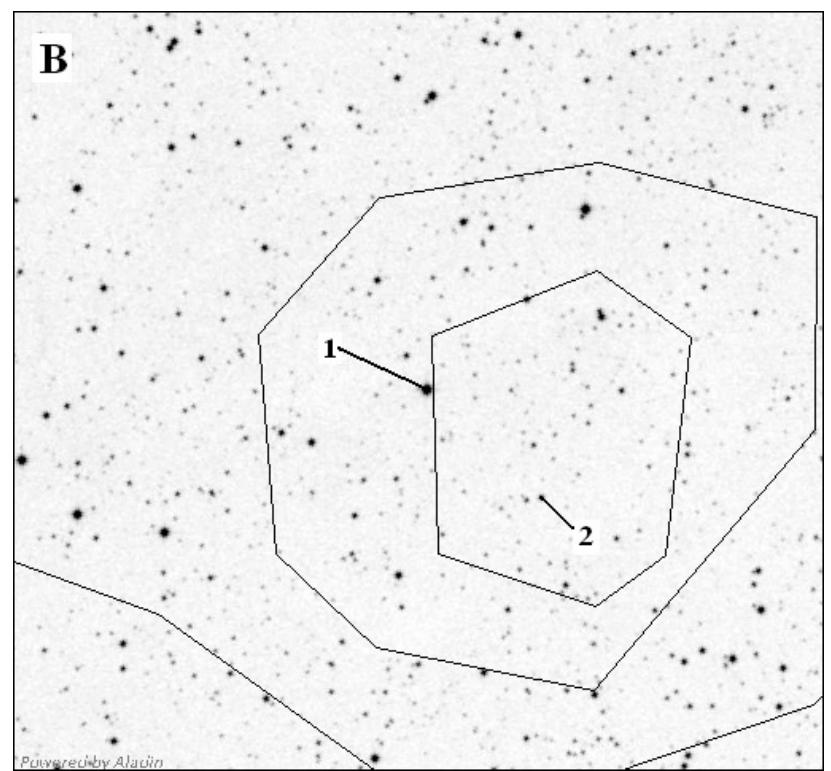

Fig. A.14. Smaller scale image of S203 (location B) with the stars 1 and 2 (image size $12.7^{\prime} \times 11.8^{\prime}$ ). 
D. Russeil et al.: Revised distances of Northern HII regions, Online Material p 10

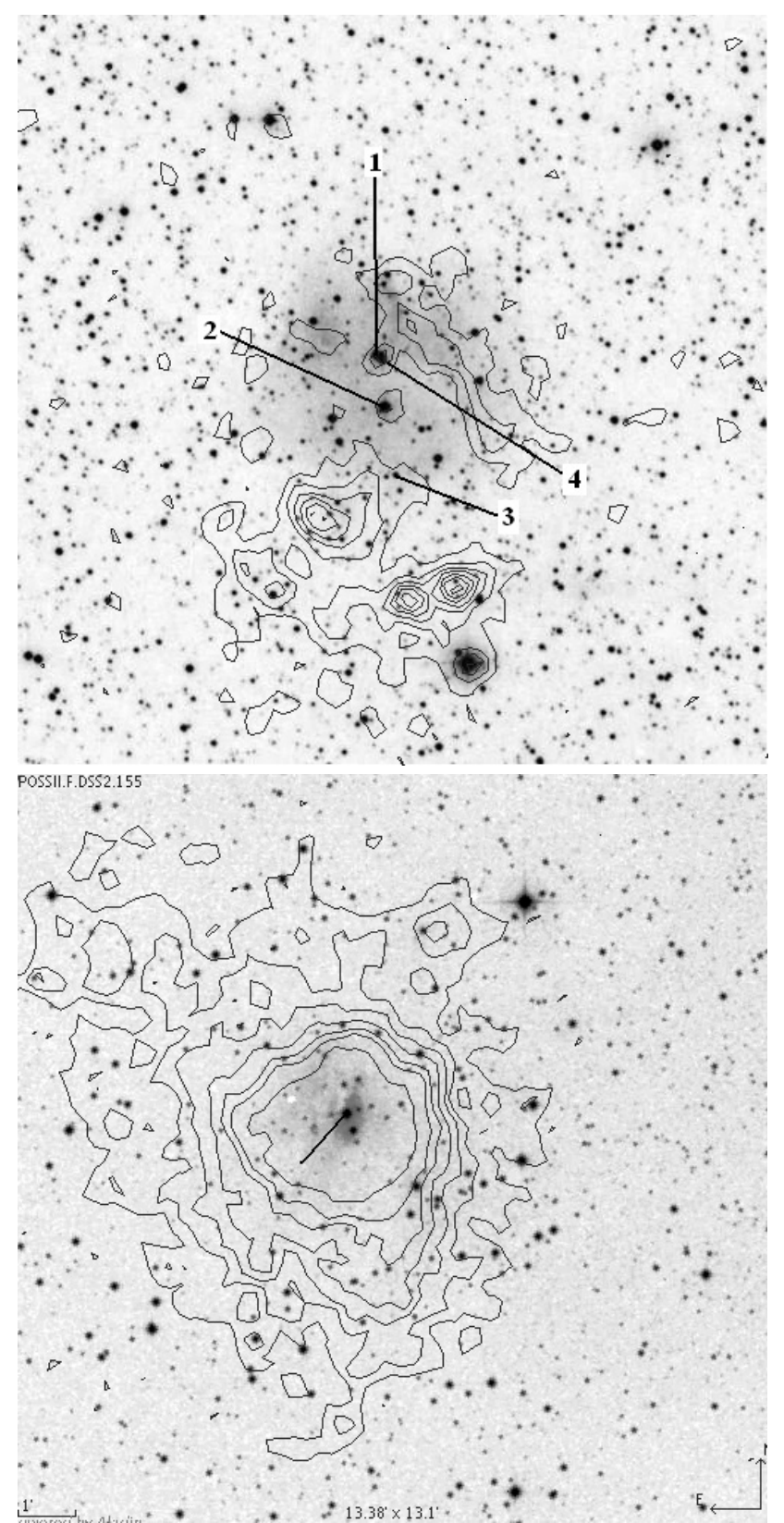

Fig. A.15. DSS images with $M S X$ band A contours of S196 (top), image size $13.8^{\prime} \times 13.9^{\prime}$ and BFS31 (bottom), image size $13.4^{\prime} \times 13.1^{\prime}$.

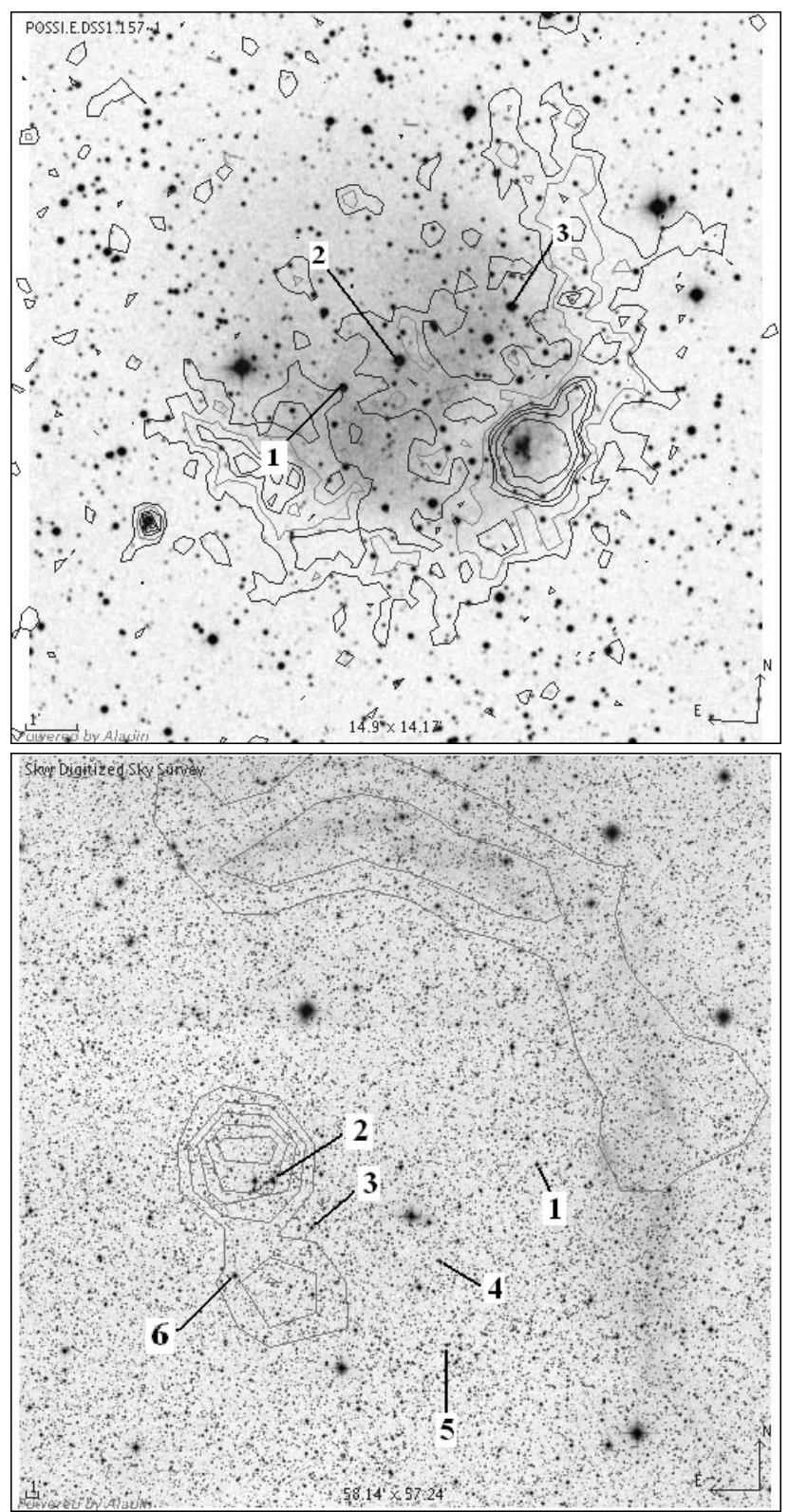

Fig. A.16. DSS image with MSX band A contours of for S217 (top), image size $14.9^{\prime} \times 14.2^{\prime}$. Bottom: DSS image of S204 (image size $58^{\prime} \times$ 57.2') with radio contours overlaid. The stars discussed in the text are 1) ALS7811, 2) ALS7833, 3) ALS7829, 4) ALS7816, 5) ALS7815 and 6) $\mathrm{BD}+56^{\circ} 866$. 
D. Russeil et al.: Revised distances of Northern HII regions, Online Material p 11

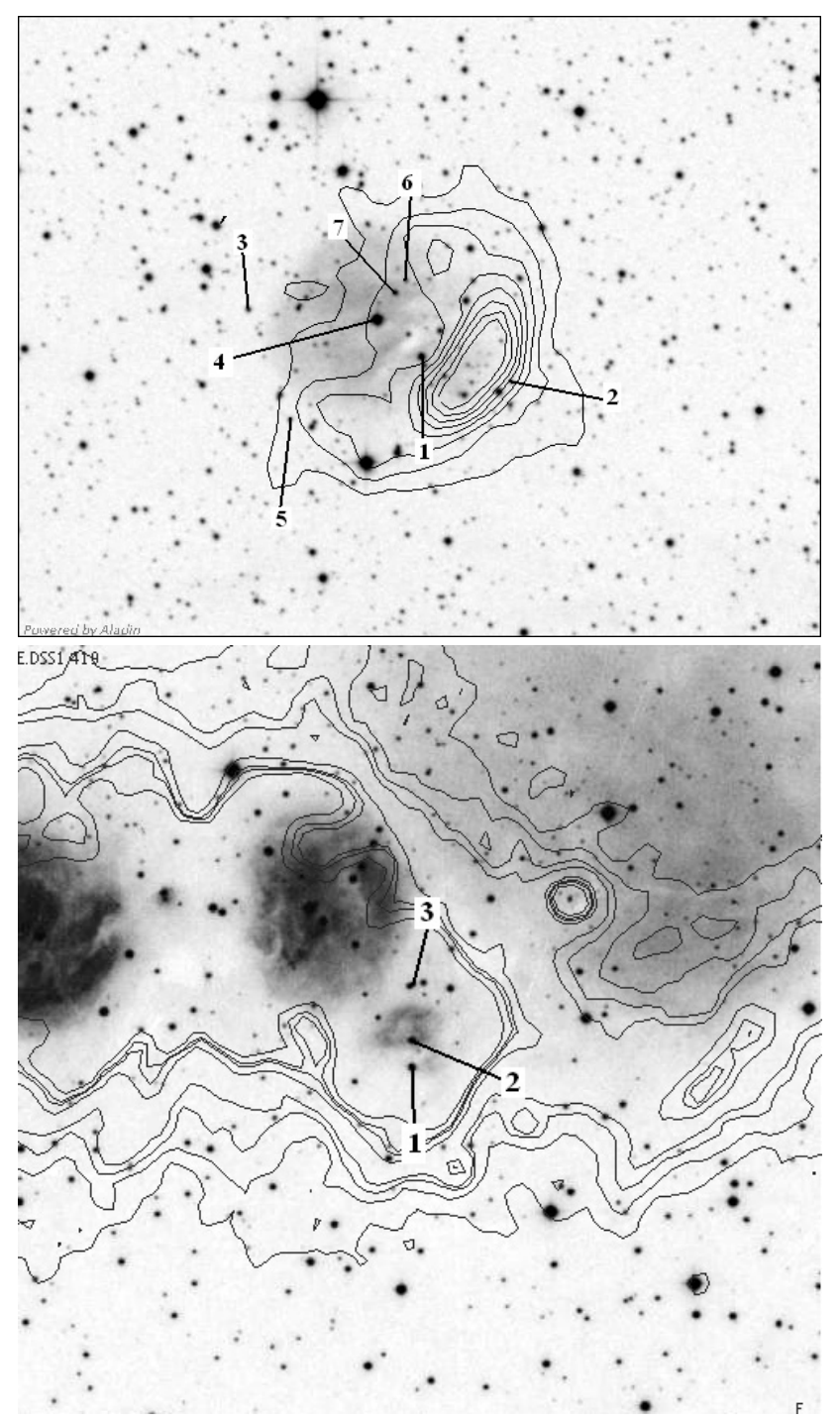

Fig. A.17. DSS images with $M S X$ band A contours of S219 (top), image size $10.1^{\prime} \times 7.8^{\prime}$ and S256 (bottom), image size $16.5^{\prime} \times 13.1^{\prime}$.

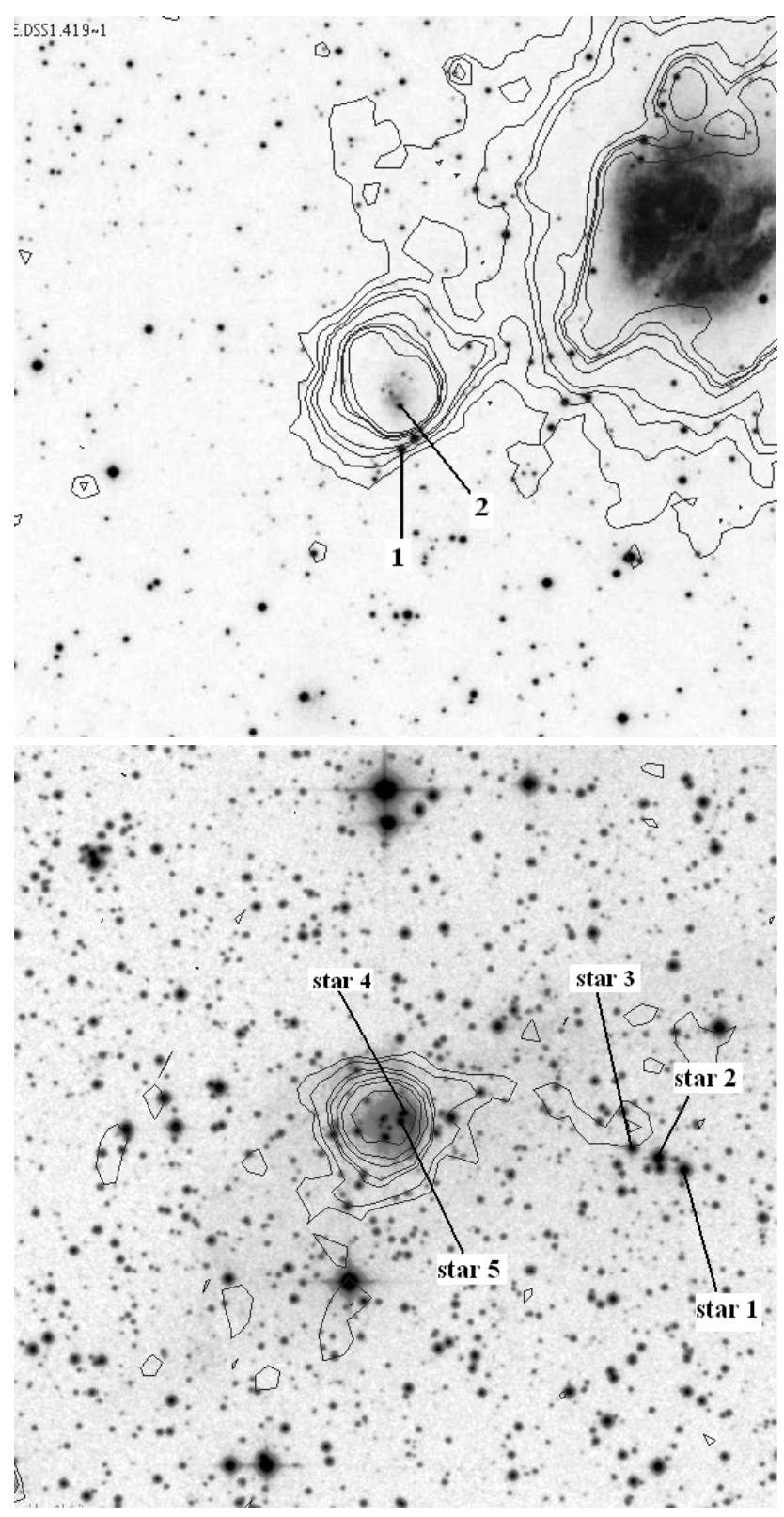

Fig. A.18. DSS images with MSX band A contours of S258 (top), image size $14^{\prime} \times 13.8^{\prime}$ and S283 (bottom), image size $9.9^{\prime} \times 9.8^{\prime}$. 
D. Russeil et al.: Revised distances of Northern HII regions, Online Material p 12

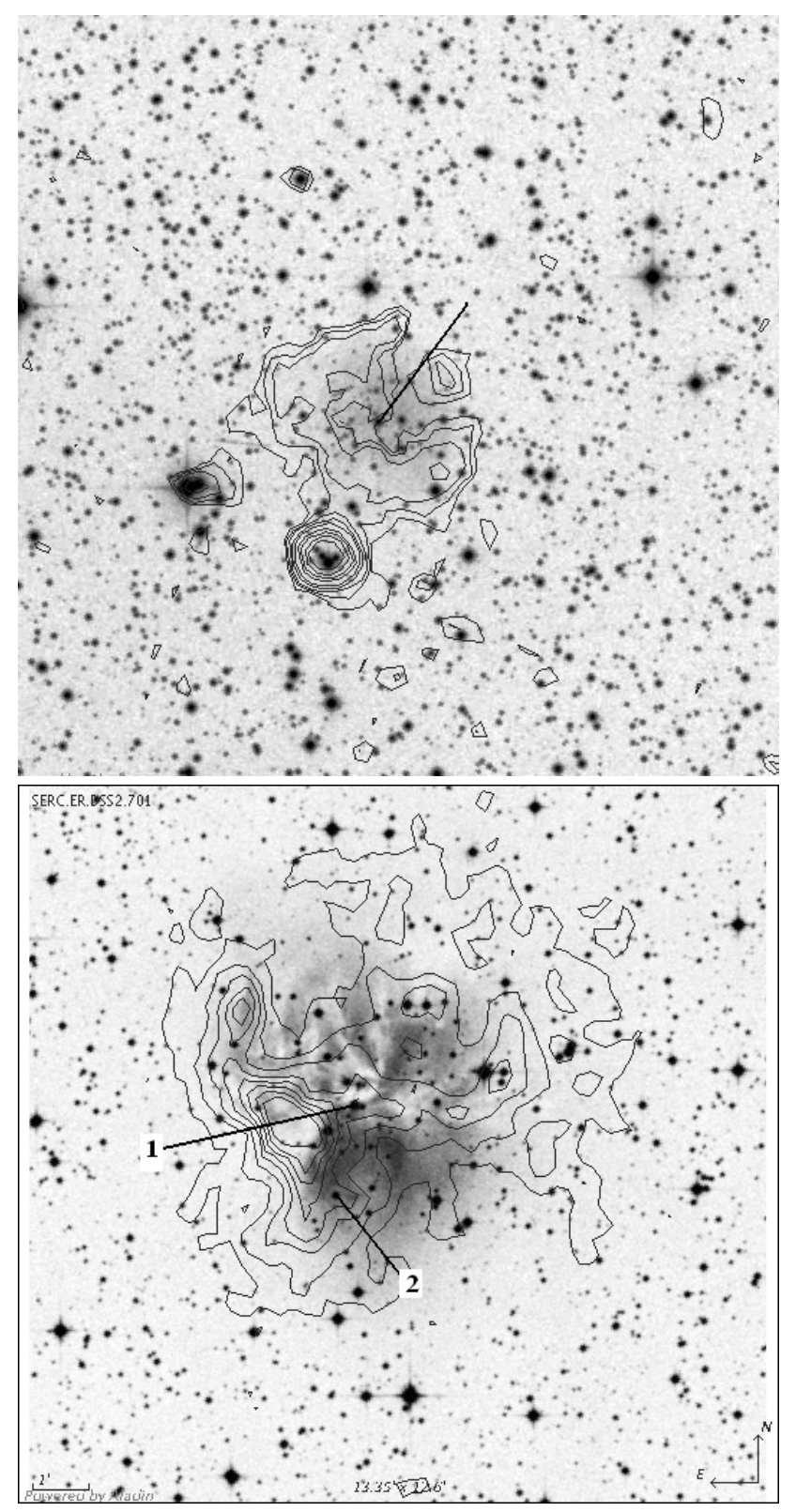

Fig. A.19. DSS images with MSX band A contours of BFS53 (top), image size $12.9^{\prime} \times 12.8^{\prime}$ and S294 (bottom), image size $13.3^{\prime} \times 12.6^{\prime}$. 
D. Russeil et al.: Revised distances of Northern HII regions, Online Material $p 13$
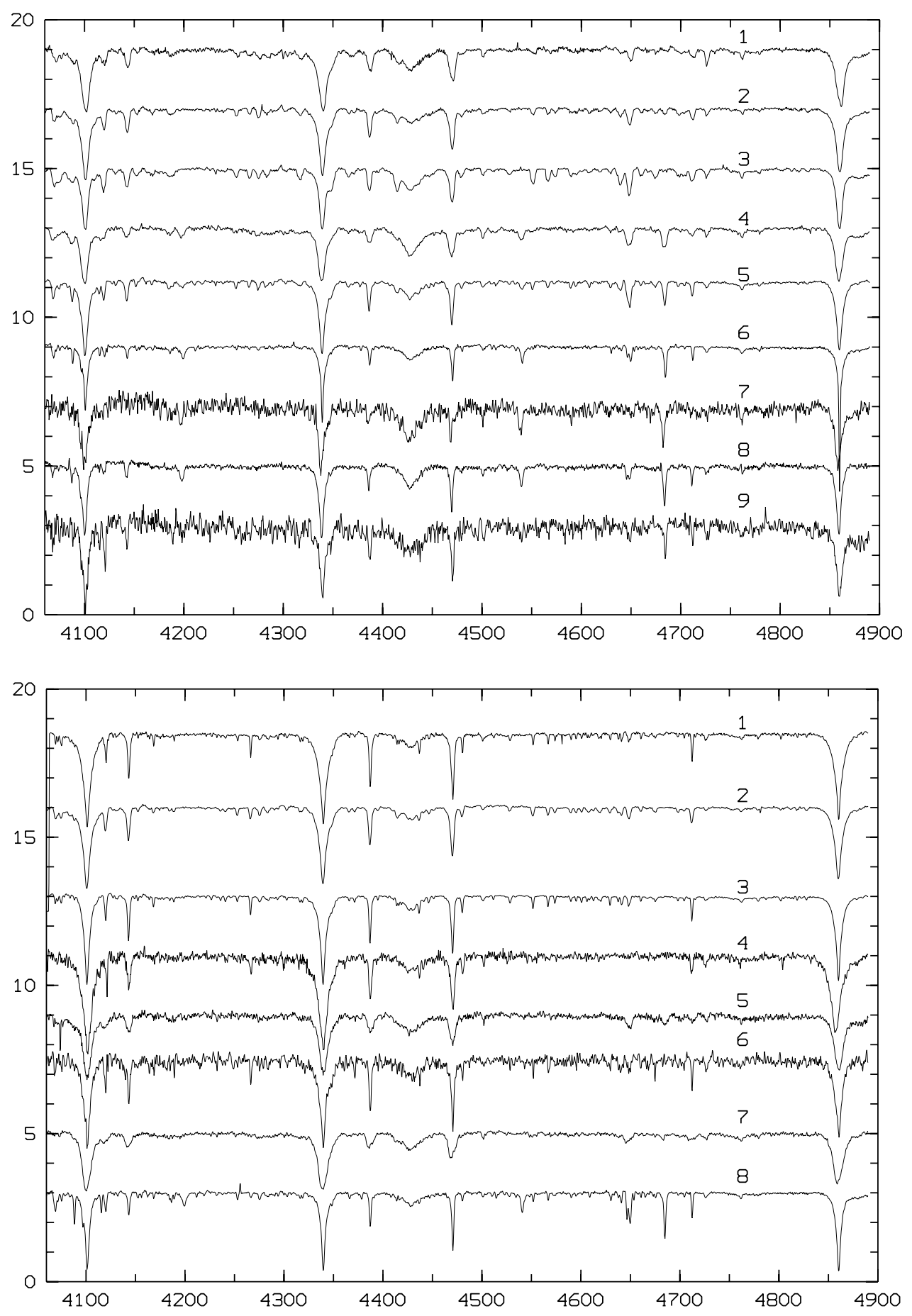

Fig. A.20. Top: 1) S175 star 1; 2) S170 star 1; 3) S163 star 1; 4) S159; 5) S153; 6) S152 star 4; 7) S141; 8) G137.8-1.0; 9) BFS10. Bottom: 1) S173 star 1; 2) S173 star 2; 3) S173 star 3; 4) S187 star 1; 5) S192 star 1; 6) S193 star 3; 7) S193 star 1; 8) S204. 
D. Russeil et al.: Revised distances of Northern HII regions, Online Material p 14
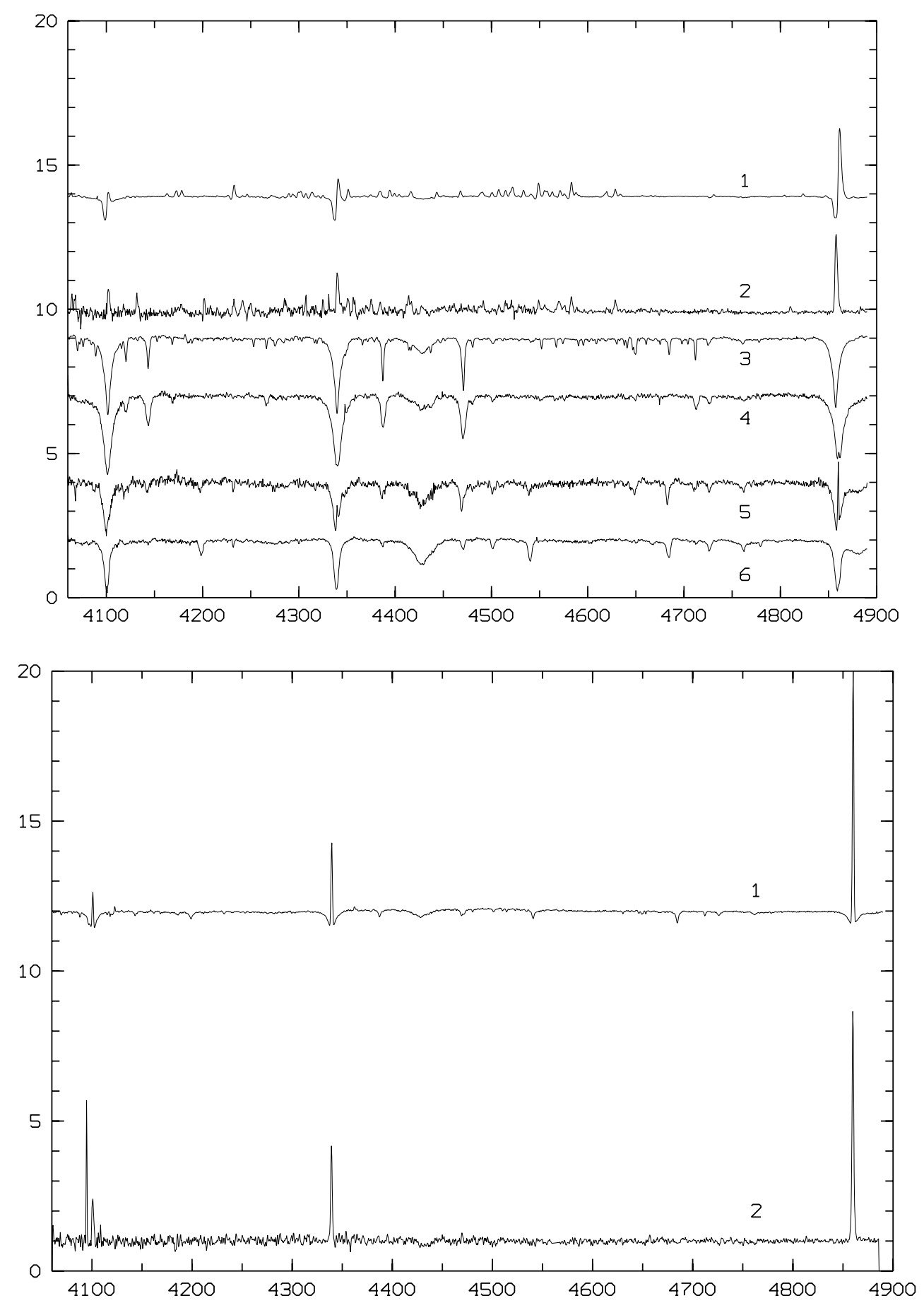

Fig. A.21. Top: 1) S182 star 1; 2) S187 star 3; 3) S219 star 4; 4) S219 star 1; 5) S158 star 2; 6) S158 star 1. Bottom: 1) S156; 2) S138 star 1. 
D. Russeil et al.: Revised distances of Northern HII regions, Online Material p 15
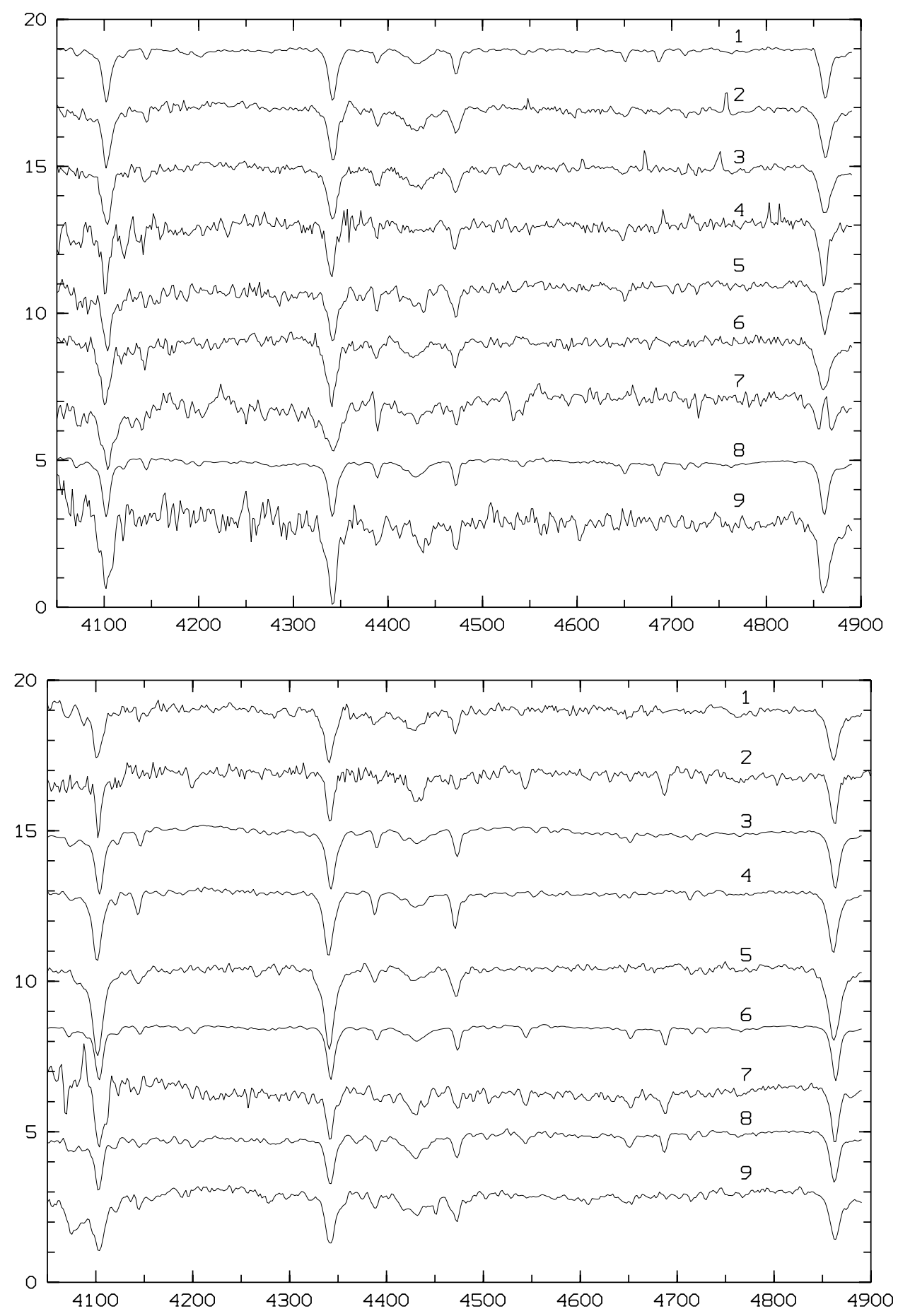

Fig. A.22. Top: 1) S258 star 2; 2) S217 star 2; 3) S203 star 3; 4) S219 star 7; 5) S256 star 2; 6) S294 star 1; 7) BFS31; 8) BFS53; 9) S196 star 4. Bottom: 1) S283 star 1; 2) BFS8 star 2; 3) S170 star 5; 4) S170 star 4; 5) S170 star 3; 6) S170 star 2; 7) S163 star 6; 8) S163 star 5 ; 9 ) S163 star 7. 
D. Russeil et al.: Revised distances of Northern HII regions, Online Material p 16

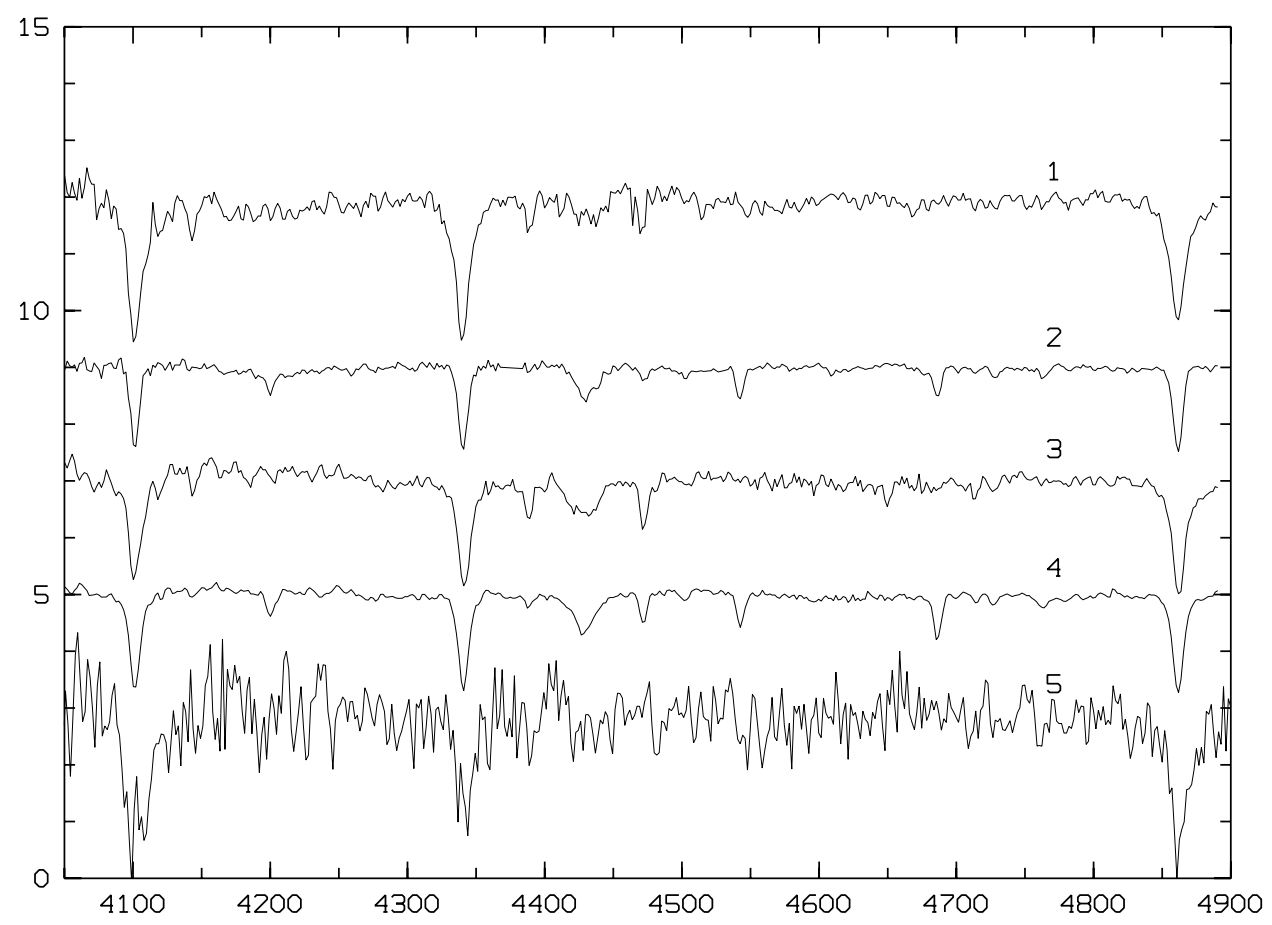

Fig. A.23. 1) DA568 star 2; 2) DA568 star 1; 3) S283 star 5; 4) S283 star 2; 5) S283 star 4. 